\title{
Study of ferritin self-assembly and heteropolymer formation by the use of Fluorescence Resonance Energy Transfer (FRET) technology.
}

Fernando Carmona ${ }^{1}$, Maura Poli ${ }^{1}$, Michela Bertuzzi ${ }^{2}$, Alessandra Gianoncelli ${ }^{2}$, Fabrizio Gangemi ${ }^{3}$ and Paolo Arosio ${ }^{1 *}$

${ }^{1}$ Molecular Biology Laboratory, Department of Molecular and Translational Medicine (DMMT), University of Brescia, Viale Europa 11, Brescia, Italy

${ }^{2}$ Proteomics Platform, Department of Molecular and Translational Medicine (DMMT), University of Brescia, Viale Europa 11, Brescia, Italy

${ }^{3}$ Laboratory of Physics, Department of Molecular and Translational Medicine (DMMT), University of Brescia, Viale Europa 11, Brescia, Italy

Running title: Ferritin self-assembly and heteropolymers formation

*To whom correspondence should be addressed: Prof. Paolo Arosio, Molecular Biology Laboratory, Department of Molecular and Translational Medicine (DMMT), University of Brescia, Viale Europa 11, Brescia, Italy. Telephone: (0039) 030394386 - (0039) 0303995.554; FAX: (0039) 030307251; Email: paolo.arosio@unibs.it. 


\begin{abstract}
Background: The high stability and strong self-assembly properties made ferritins the most used proteins for nanotechnological applications. Human ferritins are made of 24 subunits of the H- and Ltype that coassemble in an almost spherical nanocage $12 \mathrm{~nm}$ across, delimiting a large cavity. The mechanism and kinetics of ferritin self-assembly and why $\mathrm{H} / \mathrm{L}$ heteropolymers formation is favored over the homopolymers remain unclarified.

Methods: We used the Fluorescence Resonance Energy Transfer (FRET) by binding multiple donor or acceptor Alexa Fluor fluorophores on the outer surface of human $\mathrm{H}$ and $\mathrm{L}$ ferritins and then denaturing and reassembling them in different proportions and conditions.

Results: The FRET-efficiency increase from $<0.3$ of the disassembled to $>0.7$ in the reassembled allowed to study the self-assembly kinetics. We found that their assembly was complete in about one hour, and that the initial rate of self-assembly of $\mathrm{H} / \mathrm{L}$ heteropolymers was slightly faster than that of the $\mathrm{H} / \mathrm{H}$ homopolymers. Then, by adding various proportions of unlabeled $\mathrm{H}$ or L-chains to the FRET system we found that the presence of the L-chains displaced the formation of $\mathrm{H}-\mathrm{H}$ dimers more efficiently than that of the H-chains.

Conclusion: Heterodimeric (H/L) subunit association is preferred during H/L heteropolymers formation. The H-chains arrange at distant positions on the heteropolymeric shell until they reach a number above eight, when they start to co-localize and the ferroxidase activity of the heteropolymer reaches a plateau.

General significance: This favored formation of $\mathrm{H} / \mathrm{L}$ heterodimers, which is the initial step in ferritin self-assembly, contributes to explain the preferred formation of $\mathrm{H} / \mathrm{L}$ heteropolymers over the $\mathrm{H}$ or $\mathrm{L}$ homopolymers.
\end{abstract}

Keywords: Ferritin, self-assembly, heteropolymers, homopolymers, fluorescence energy transfer (FRET)

Abbreviations: FTH, homopolymer of human ferritin H-chains; FTL, homopolymer of human ferritin L-chains; FTHL, heteropolymer of human $\mathrm{H}$ - and L-chains; FTMT, homopolymer of human mitochondrial chains; AF, alexa fluor; FTH-488, full-labeled FTH with AF488; FTH-555, full-labeled FTH with AF555; FTL-555, full labeled FTL with AF555; H-488, H-chain labeled with AF488; H555, H-chain labeled with AF555; L-555, L-chain labeled with AF555; FRET, fluorescence resonance energy transfer; PAGE, polyacrylamide gel electrophoresis: MALDI-TOF, Matrix-Assisted Laser Desorption/Ionization Time-of-Flight Mass Spectrometry. 


\section{Introduction}

Most multimeric proteins have the capacity to self-assemble and form functional multisubunit protein complexes by spontaneous association of different subunits through multiple noncovalent interactions. The mechanism of self-assembly is not fully understood, and can be analyzed by the study of simple models. Ferritin is one of these models, and in fact it has properties that have been largely exploited in nanotechnology. Ferritin comprises 24 similar subunits that assemble into an almost spherical shell to form a dodecahedron characterized by a 432-point symmetry $12 \mathrm{~nm}$ in diameter (1). Each subunit is composed of a four-helix bundle, with a long loop between helices B and C, and a fifth short C-terminal helix E. This basic structure is characteristic, unique and conserved in all ferritin types (2). In the 24-mer structure each subunit is flanked by 6 subunits. One ferritin major property is that it can be easily produced in a recombinant form as a highly stable 24-mer protein that can be readily denatured to produce free subunits, and those under proper conditions quantitatively self-assemble to reform the stable $24-$ mer shell. This process has been exploited to encapsulate in the cavity molecules of pharmacological and chemical interest $(3,4,5)$. This reaction, although highly used has been analyzed in detail only in a few reports $(6,7,8)$ and the kinetics of the reassembly have been studied in detail only for ferritins of bacterial origin by using X-ray scattering (9).

An interesting property of ferritin that has been largely overlooked is the capacity to form heteropolymers made of different proportions of two or more subunit types on the same shell. In particular, the mammalian ferritins are made of two subunit types, $\mathrm{H}$ and $\mathrm{L}$, that, although being synthetized from different genes, quantitatively coassemble to form hybrid shells without the accumulation of $\mathrm{H}$ and $\mathrm{L}$ homopolymers $(10,11)$. Interestingly, this occurs also in vitro, and despite the evidence that L-chain homopolymers have a higher stability than the H-chain ones, when the two chains are renatured together they form heteropolymers in which the ratio between the two subunits is dictated only by their availability. Thus, it is possible to produce ferritin shells ranging from $\mathrm{L}_{24} \mathrm{H}_{0}$ to $\mathrm{L}_{0} \mathrm{H}_{24}$ with all the intermediates $(12,13)$. The mechanism of this reaction of assembly and how the two subunits preferentially interact to form the hybrid 24-mer is unclear. In particular, it would be of interest to verify if their distribution in the 24-mer structure is random or it follows a specific pattern directed by specific interactions. The 24-mer ferritin can be disassembled by using extreme $\mathrm{pH}$ values $(<2$ or $>11)$ and then renatured by returning to neutral $\mathrm{pH}(14)$, or it can be fully denatured in acidic guanidine hydrochloride and renatured by dilution in renaturing buffer $(13,15)$. The pathway of assembly has been initially studied using horse spleen ferritin, suggesting a path with the formation of dimers followed by tetramers and other oligomers (16). A study using recombinant $\mathrm{H}$-chain ferritin mutants supported the formation of the dimers in the first step of the assembly, followed by aggregation around either the 3-fold or the 4-fold axis (17). Recently, that subunit dimers are the first intermediates in the self-assembly pathway was supported by using a new technique in which the interaction of the subunit dimers was engineered to make it copper-dependent (18). Ferritin heteropolymers can also be made by expressing the two chains inside the E. coli, and the rate of expression of each chain determines their molar ratio in the final protein shell, without the formation of homopolymers (19). However, it remains to be clarified whether, during the formation of heteropolymers, the intermediates are subunit heterodimers or homodimers. The understanding of the heteropolymers formation may be also of nanotechnological interest as it would permit to predict how the specific properties attached to the two chains are distributed within a 24-mer molecule, if they may be contiguous or separated, and be exploited for the introduction of multiple functionalities in a single molecule.

Qualitative analysis of protein-protein interactions in vitro is of fundamental importance in understanding these complex biochemical processes. Among several methods that are available in this context, Fluorescence Resonance Energy Transfer (FRET) technique has been widely used in vitro and in vivo to study protein-protein interactions $(20,21,22,23,24)$. Since FRET efficiency is proportional to $1 / \mathrm{r}^{6}$, where " $\mathrm{r}$ " is the distance between the donor and the acceptor, the FRET signal provides a high degree of spatial sensitivity between 2 and $12 \mathrm{~nm}(7)$.

In the present study, we have analyzed the in vitro assembly of ferritin homopolymers made of the human $\mathrm{H}$-chain and of $\mathrm{H} / \mathrm{L}$ heteropolymers after denaturing the ferritin in acidic guanidine $\mathrm{HCl}$ and renaturing by dilution into renaturation buffer. Labeling the subunits with FRET donor and 
acceptor fluorophores allowed to track the reassembly process. The results show that the reassembly reaction has a half time of 30-40 min, being faster for the $\mathrm{H} / \mathrm{L}$ heteropolymers than for the $\mathrm{H}$ homopolymers. Moreover, the study of the effect of various proportions of unlabeled H- and L-chains in the energy transfer indicated that the preferential formation of $\mathrm{H} / \mathrm{L}$ heteropolymers versus the corresponding homopolymers is mainly due to the preferential formation of $\mathrm{H} / \mathrm{L}$ heterodimers over homodimers during the process of self-assembly. Finally, we found that the H-chains arrange at distant positions on the heteropolymeric ferritin shell until they reach a number above eight, when they start to co-localize.

\section{Materials and methods}

\subsection{Recombinant human H-ferritin (FTH), L-ferritin (FTL) and Mitochondrial ferritin (FTMT) preparation.}

Recombinant human FTH, FTL and FTMT were prepared as described by Santambrogio et al. (2000) (25). Briefly, homopolymers of human H- and L-chains were expressed by E. coli strains transformed with the pET12b (FTH and FTMT) and pDS20pTrp (FTL) vectors, which encode for the full and correct amino acid sequences of the chains. For FTH and FTMT expression, E. coli strain was grown at $37^{\circ} \mathrm{C}$ in LB broth and expression induced by $1 \mathrm{mM}$ IPTG for 3 hours. For FTL expression, under the Trp promoter, E. coli strain B was grown at $37^{\circ} \mathrm{C}$ in $\mathrm{M} 9$ broth for $7 \mathrm{~h}$. The cells were harvested and disrupted by sonication, and the soluble homogenates were heated at $75^{\circ} \mathrm{C}$ for $10 \mathrm{~min}$, precipitated with ammonium sulfate $(520 \mathrm{~g} / \mathrm{liter})$, and treated with DNase. The final purification steps consisted of gel filtration on a Sepharose 6B column for FTH and FTMT and of gel filtration on Sephacryl S-200 for FTL, followed by a DEAE column. All ferritins were electrophoretically pure. Protein concentration was determined with BCA reagent (Pierce) using bovine serum albumin as standard.

\subsection{Cysteine labeling on FTH.}

Human FTH homopolymer was fluorescently labeled at Cys90 using Alexa Fluor $488 \mathrm{C}_{5^{-}}$ maleimide (Molecular Probes). Briefly, human FTH $(10 \mu \mathrm{M})$ in $20 \mathrm{mM}$ Tris buffer $\mathrm{pH} 7.5$ was reacted with a 400 -fold molar excess of Alexa Fluor $488(4 \mathrm{mM})$ during 3 hours, in the dark at room temperature under gentle agitation. After the labeling reaction, unreacted dye was removed by exhaustive dialysis (MWCO $12 \mathrm{kD}$ ) using a high retention seamless cellulose tubing membrane (Sigma) followed by Sephadex G-25 desalting column (GE Healthcare) and further purified by gel filtration chromatography on a Superose-12 column (GE Healthcare).

\subsection{Lysine labeling on FTH and FTL.}

Human FTH and FTL homopolymers were fluorescently labeled at Lys86/87 using Alexa Fluor 555 NHS-ester (Molecular Probes). Prior to the labeling reaction, FTH and FTL were buffer exchanged to $50 \mathrm{mM}$ sodium bicarbonate buffer at $\mathrm{pH} 8.5$, and then reacted with a 400 -fold molar excess of Alexa Fluor $555(4 \mathrm{mM})$ during 3 hours, in the dark at room temperature under gentle agitation. Unreacted dye was removed as described for FTH labeling with AF488.

\subsection{FTH-488, FTH-555 and FTL-555 characterization.}

The absorbance of the collected chromatographic fractions was read at $280 \mathrm{~nm}$ (protein) and at 495 or $555 \mathrm{~nm}$ for Alexa Fluor 488 or 555, confirming the co-elution of protein and dye. Nondenaturing $7 \%$ gel electrophoresis (PAGE) scanned under UV light prior to Coomassie staining showed the co-migration of protein and fluorophore and thus confirmed the covalent binding of the Alexa Fluor to the ferritins. Fluorophore to protein $(\mathrm{F} / \mathrm{P})$ molar ratios were calculated by UV/vis spectroscopy using the known extinction coefficients of the proteins at $280 \mathrm{~nm}$ (FTH: $\varepsilon^{280}=505,000$ $\mathrm{M}^{-1}$; FTL: $\varepsilon^{280}=456,000 \mathrm{M}^{-1}$ ), and the fluorophores (AF488: $\varepsilon^{495}=71,000 \mathrm{M}^{-1}$; AF555: $\varepsilon^{555}=$ $150,000 \mathrm{M}^{-1}$ ). Absorbance contributions at $280 \mathrm{~nm}$ from the fluorophores were subtracted following 
the instructions brovided by manufacturer (https://tools.thermofisher.com/content/sfs/manuals/mp10168.pdf). Fluorophore/Protein values of $21.7 \pm 0.3,19.1 \pm 0.6$ and $20.1 \pm 0.5$ for FTH-488, FTH-555 and FTL-555 respectively, were obtained at triplicate samples.

2.5. Matrix-Assisted Laser Desorption/Ionization Time-of-Flight Mass Spectrometry [MALDI-TOF MS] analysis.

MALDI-TOF-MS analysis was performed on AB Sciex 5800 MALDI-TOF/TOF-MS in linear ion mode using the matrix SUPER-2,5-Dihydroxybenzoic acid (Super-DHB, Sigma) as described by Gianoncelli et al. (26).

\subsection{FRET analysis.}

In our FRET system, AF488 $\left(\lambda_{\text {ex }}=480 \mathrm{~nm} ; \lambda_{\text {em }}=520 \mathrm{~nm}\right)$ served as the donor while AF555 $\left(\lambda_{\mathrm{ex}}=540 \mathrm{~nm} ; \lambda_{\mathrm{em}}=570 \mathrm{~nm}\right)$ served as the acceptor. Fluorescence measurements were carried out on a multimode plate reader (EnSight, Perkin Elmer) under $480 \mathrm{~nm}$ excitation wavelength using 100 excitation flashes and $1 \mathrm{~nm}$ slit widths. For each sample two fluorescence emission spectra were recorded: (1) the sample was excited at $480 \mathrm{~nm}$ (donor excitation) and emitted light was collected from $500-700 \mathrm{~nm}$ at $1 \mathrm{~nm}$ increments; and (2) the sample was excited at $540 \mathrm{~nm}$ (acceptor excitation) and emitted light was collected from 555-700 $\mathrm{nm}$ at $1 \mathrm{~nm}$ increments. Experiments were replicated at least three times, and the presented data are representative results unless stated otherwise. Efficiency of energy transfer was reported as (ratio) ${ }_{\mathrm{A}}$ whereby the acceptor (AF555) fluorescence via energy transfer is normalized against acceptor fluorescence via direct excitation. (ratio) A $_{\text {parameter is directly }}$ proportional to FRET efficiency, and changes in (ratio) A $_{\mathrm{A}}$ across different experimental conditions serve as a proxy for conformational changes studies $(30,35,36)$. For each FRET construct, a donoronly (FTH-488) sample was prepared and its emission spectrum after $480 \mathrm{~nm}$ excitation collected. This spectrum was normalized to and subtracted from each experimental emission spectrum to generate an extracted fluorescence spectrum for the acceptor via energy transfer. The integrated area under this curve from 520-700 $\mathrm{nm}$ was calculated and divided by the integrated area under the curve of a spectrum resulting from direct acceptor (FTH-555 or FTL-555) excitation at $540 \mathrm{~nm}$, resulting in $(\text { ratio) })_{\mathrm{A}}$, i.e. the enhancement of acceptor fluorescence due to FRET (Supplementary S1B). Predicted FRET efficiencies at each subunit distance were calculated using the equation: $E=R_{0}{ }^{6} /\left(R_{0}{ }^{6}+r\right)$ (Eq. 1 ), where $E$ denotes the efficiency of FRET, $R_{0}$ is the theoretical Förster distance for the fluorophore couple (70 $\AA$ ) and $r$ the distance between the donor and the acceptor (27).

\subsection{Ferritin disassembly and reassembly.}

Ferritin disassembly and reassembly reactions were carried out as described by Santambrogio and co-workers (13). Briefly, disassembly of the ferritin homopolymers was carried out by 18-hour incubation in $6 \mathrm{M} \mathrm{Gdn}-\mathrm{HCl} \mathrm{pH} 3.5$ for $\mathrm{FTH}$ and $8 \mathrm{M} \mathrm{Gdn}-\mathrm{HCl} \mathrm{pH} 3.5$ for FTL at $4^{\circ} \mathrm{C}$. Unfolded chains were then mixed at the desired proportions for $30 \mathrm{~min}$ and reassembled back to the 24-meric ferritin structure by 10-fold dilution with $0.1 \mathrm{M}$ Tris- $\mathrm{HCl} \mathrm{pH}$ 7.5, $2 \mathrm{mM}$ TCEP. Samples were let refold for 24 hours at $4^{\circ} \mathrm{C}$.

\subsection{FRET-based detection of ferritin assembly.}

Energy transfer was analyzed firstly on reassembled ferritins containing an equimolar amount of donor and acceptor chains. To do so, full-labeled ferritin homopolymers (FTH-488, FTH-555 and FTL-555) were disassembled as described above and mixed in a 1:1 proportion of unfolded chains $(\mathrm{H}-488: \mathrm{H}-555$ or $\mathrm{H}-488: \mathrm{L}-555$, total protein $0.1 \mu \mathrm{M})$ at $4^{\circ} \mathrm{C}$ in the darkness. After $30 \mathrm{~min}$, the denatured mixture was divided in two: one half was 10-fold diluted in $6 \mathrm{M}$ Guanidine Hydrochloride $\mathrm{pH} 7.5$ to leave the chains disassembled, and the other half reassembled to the 24-mer structure as described before to produce the corresponding full-labeled homopolymers (FTH-488/555, $0.01 \mu \mathrm{M})$ 
and heteropolymers (FTHL-488/555, $0.01 \mu \mathrm{M}$ ) containing both fluorophores at equimolar proportion. All samples were prepared in triplicate and allowed to reach the equilibrium for 48 hours before being analyzed. After 48 hours, emission spectra of 1) the reassembled full-labeled ferritins (FTH-488/555 or FTHL488/555); 2) the 1:1 mixture of disassembled chains (H-488:H-555 or H-488:L-555) as well as 3) the 1:1 mixture of the native FTH-488 and FTH-555 homopolymers, were recorded under 480 $\mathrm{nm}$ excitation and (ratio) $)_{\mathrm{A}}$ calculated as described before.

\subsection{Kinetics of ferritin self-assembly in homopolymers and heteropolymers.}

The kinetics of ferritin self-assembly were studied by FRET monitoring the emission spectra of reassembled ferritins immediately after dilution in the renaturation buffer. Equimolar mixtures of unfolded H-488+H-555 chains to yield homopolymers and equimolar mixtures of unfolded $\mathrm{H}-488+\mathrm{L}-$ 555 for heteropolymers, were reassembled at a final concentration of $0.01 \mu \mathrm{M}$ and their emission spectra under $480 \mathrm{~nm}$ excitation immediately recorded every $30 \mathrm{~s}$ during the first 3 hours and then every hour until a total of 24 hours. In parallel, controls containing the unfolded mixtures $(\mathrm{H}-488+\mathrm{H}-$ 555 ; H-488+L-555) as well as equimolar mixtures of native homopolymers (FTH-488+FTH-555; FTH-488+FTL-555) at similar concentrations than that of the reassembled, were also monitored on time. All samples were prepared and monitored by triplicate. At different times of reaction $(5,30,120$ and $1440 \mathrm{~min}$ ), the extent of assembly onto the 24-meric structure was analyzed using $7 \%$ nondenaturing gel electrophoresis in the presence of $0.2 \%$ SDS, to inhibit the assembly of the subunits, but not enough to disrupt the already formed structure.

\subsection{Preparation of full-labeled ferritins containing different donor:acceptor ratios.}

To synthesize full-labeled ferritins containing different proportions of donor:acceptor subunits on their structures $(5: 1,2: 1,1: 1,1: 2,1: 5)$, a batch of unfolded chain mixtures containing a fixed concentration of H-488 $(0.06 \mu \mathrm{M})$ and increasing proportions of $\mathrm{H}-555$ chains $(0.012,0.03$, $0.06,0.12$ and $0.3 \mu \mathrm{M}$ ) was prepared and then reassembled following the above described protocol. Non-denaturing 7\% gel electrophoresis scanned with UV light prior to Coomassie staining confirmed the reassembly of the homopolymers at the pre-incubated proportions as shown by the ladder pattern of electrophoretic migration. Similar protocol was followed but titrating with FTL-555 instead of FTH-555 to produce the corresponding heteropolymers (FTHL488/555). All samples were done by triplicate and allowed to reach the equilibrium for 48 hours before being analyzed.

\subsection{Design of a FRET system capable to track subunit distances on ferritin shells.}

FRET-efficiency is strongly dependent on the distances between a donor-acceptor FRET pair. Indeed, the distance between donor and acceptor dyes can be directly calculated from the FRET efficiency, but accurately relating these variables requires knowledge of numerous complex parameters (36). Without pursuing a more rigorous calculation, we designed a system capable to compare the effect of H- and L-chains in the assembly of the H-chains. More specifically, we used donor:acceptor H-chain couples $(\mathrm{H}-488+\mathrm{H}-555)$ as a sensor of proximity when co-assembled with unlabeled $\mathrm{H}$ - and L-chains. To do so, unlabeled $\mathrm{H}$-chains were titrated at different proportions with a mixture containing an equimolar amount $(\mathrm{H}-488+\mathrm{H}-555)$ chains. We co-assembled 1 couple of labeled $\mathrm{H}(1 \mathrm{H}-488+1 \mathrm{H}-555)$ with 22 unlabeled ones, then 2 couples of labeled $\mathrm{H}(2 \mathrm{H}-488+2 \mathrm{H}-555)$ with 20 unlabeled ones, three copies $(3 \mathrm{H}-488+3 \mathrm{H}-555)$ with $18 \mathrm{H}$, four copies $(4 \mathrm{H}-488+4 \mathrm{H}-555)$ with $16 \mathrm{H}$, five copies $(5 \mathrm{H}-488+5 \mathrm{H}-555)$ with $14 \mathrm{H}$, and six copies $(6 \mathrm{H}-488+6 \mathrm{H}-555)$ with $12 \mathrm{H}$. Similar was done but using not-labeled L-chains titrated with the donor:acceptor H-chain couples $(\mathrm{H}-488+\mathrm{H}-$ 555), to yield the corresponding $\mathrm{H} / \mathrm{L}$ heteropolymers containing increasing amounts of fluorescent $\mathrm{H}$ subunits. Composition of the produced ferritins are listed in Table 1.

\subsection{Iron uptake.}

The kinetics of iron uptake were studied as described in (13) using a molar ferritin:iron ration of 1:1000. A freshly prepared $100 \mathrm{mM}$ solution of ferrous ammonium sulfate in water was added to 
$0.1 \mu \mathrm{M}$ apoferritins $\left(50 \mu \mathrm{g} / \mathrm{mL}\right.$ in $0.1 \mathrm{M}$ Tris- $\mathrm{HCl}$ buffer $\mathrm{pH} 6.5$ at $25{ }^{\circ} \mathrm{C}$ to a final $0.1 \mathrm{mM}$ concentration. The formation of the amber iron product was monitored by the increase of absorbance at $310 \mathrm{~nm}$.

\section{Results}

\subsection{Labeling of $H$ and L ferritin homopolymers for FRET analysis.}

We initially explored the possibility to label specific sites on the outer surface of assembled ferritin shells by using activated fluorophores. Lysine and cysteine are easily labeled amino acids and human ferritin H-chain has 13 Lys and 3 Cys, of which only Lys86, Lys87 and Cys90 are exposed at the outer surface of the 24-meric shell and thus accessible to solvent. On the other hand, the human Lchain has 11 Lys and 1 Cys, of which only the Lys86 and Lys87 (H-chain numbering) are exposed at the outer surface and accessible on the 24-meric structure. These residues are placed at the long loop connecting helix $\mathrm{B}$ and $\mathrm{C}$ of each subunit (Fig. 1A). We measured in silico the inter-chain interresidue distances between Cys90 and Lys86/Lys87 on an assembled shell, finding that the shortest one $(2.7 \mathrm{~nm})$ is to the adjacent subunit along the 2 -fold axis of a subunit-subunit dimer, and the ones to the other five adjacent subunits range from 4.4 to $7.0 \mathrm{~nm}$ (Fig. 1B). The optimal distance between fluorophores for FRET measurements ranges from 2 to $12 \mathrm{~nm}$ and the efficiency of energy transfer decreases as $1 / \mathrm{r}^{6}$, where " $\mathrm{r}$ " is the distance between the fluorophores $(27,28)$. Given this fact, the monitored energy transfer is an average dominated by the closest tags. We chose to use Alexa Fluor 488 as FRET donor and Alexa Fluor 555 as FRET acceptor, a conjugate couple largely used $(29,30,31)$. FRET between this fluorophore pair is efficient in this context since the distance for $50 \%$ FRET (Förster distance, $R_{\mathrm{o}}$ ) is $7.0 \mathrm{~nm}$, compatible with the range 2.7 to $7.0 \mathrm{~nm}$ indicated above. Thus, in our system the obtained FRET signal from conjugate fluorophores on Cys90 in one subunit and Lys86/87 in other subunit would arise mainly from the interaction along the 2-fold axis in the formation of subunit dimer, which is considered the first and key intermediate in the self-assembly pathway $(6,32,33,18)$. To this aim, we reacted FTH homopolymer with Alexa Fluor $488 \mathrm{C}_{5}$ maleimide to label Cys90 and obtain FTH-488, and Alexa Fluor 555-succinimidyl ester with FTH homopolymer to label Lys86/87 and obtain FTH-555. In a typical experiment, the FTH homopolymeric shell was reacted with an excess of fluorophore for 3 hours at room temperature, exhaustively dialyzed to remove unbound dye and then subjected to gel filtration chromatography. The covalent binding of the Alexa Fluor to ferritin was verified by the co-elution of protein and fluorophore from the column and by the florescence associated with the protein band of assembled ferritins on non-denaturing PAGE (Fig. 2, $A$ and $B$ ). Fluorophore conjugation did not cause aggregation or denaturation, but increased the electrophoretic mobility, consistent with the charge of the bound molecules (Fig. 2B). From the UV-Vis absorption spectra (Fig. S1A), the Fluorophore/Protein molar ratios were calculated to be $21.7 \pm 0.3$ for FTH-488 and $19.1 \pm 0.6$ for FTH555 despite the presence of two accessible Lys, suggesting that the number of fluorophores covalently bound to exposed Lys or Cys accounted to about one per ferritin subunit. We also treated the FTL homopolymeric shell with Alexa Fluor 555-succinimidyl ester to label Lys86/87 and obtain FTL-555 using the same procedure as for FTH. This resulted in the binding of $20.1 \pm 0.5$ fluorescent tags per ferritin shell, as for FTH, despite the presence of two accessible Lys suggesting that only one of the adjacent Lys was labeled under these conditions. To verify this, we performed qualitative MALDITOF mass spectrometry analyses on FTL-555, FTH-488 and FTH-555. The spectra showed for each protein the presence of non-labeled species accompanied by a single heavier species with a molecular weight difference corresponding with that of a single fluorophore (Fig. S2, and Table S2). Thus, we concluded that under this labeling conditions only one of the adjacent Lys was labeled. The normalized absorption and emission spectra of FTH-488 $\left(\lambda_{\text {ex }}=488 \mathrm{~nm}, \lambda_{\text {em }}=520 \mathrm{~nm}\right)$ overlaps at $\sim 520 \mathrm{~nm}$ to those of FTH-555 $\left(\lambda_{\mathrm{ex}}=555 \mathrm{~nm}, \lambda_{\mathrm{em}}=570 \mathrm{~nm}\right)$ showing that both probes can serve as a donor/acceptor pair in FRET experiments (Fig. 2C). FTL-555 absorption and emission spectra behaved similarly than those of FTH-555 (not shown).

\subsection{FRET-based detection of ferritin assembly.}


Our aim was to study ferritin self-assembly using FRET following the scheme illustrated in Figure 3A. The ferritins labeled with the FRET-donor (FTH-488) and FRET-acceptor (FTH-555) were disassembled, mixed at proper proportions and the reassembly monitored by FRET. In initial experiments, we disassembled the ferritins by incubation at $\mathrm{pH} 2.0$ and reassembly by $\mathrm{pH}$ jump to $\mathrm{pH}$ 7.4, but irreversible quenching of fluorescence was observed after incubation at acidic $\mathrm{pH}$, probably due to chemical modification of the fluorescent probes, which are reported to be stable at $\mathrm{pH}$ ranges between 3 and 10 (34). Better results were obtained by denaturing FTH in $6 \mathrm{M}$ guanidine $\mathrm{HCl}$ at $\mathrm{pH}$ 3.5 for $18 \mathrm{~h}$, and then renaturing it by 10 -fold dilution in $0.1 \mathrm{M}$ Tris- $\mathrm{HCl} \mathrm{pH} \mathrm{7.5,} \mathrm{following} \mathrm{the}$ protocol described by Santambrogio et al. (1993) (13). Thus, to verify if the co-assembly of subunits carrying the FRET donor and acceptor produced evident FRET signal, FTH-488 and FTH-555 homopolymers were denatured separately, mixed in 1:1 proportion and then reassembled back to the 24-meric structure. After $48 \mathrm{~h}$, emission spectra under direct excitation of the donor at $480 \mathrm{~nm}$ were collected. Figure $3 B$ shows that the spectrum of the 1:1 mixture of the native FTH-488 and FTH-555 shells is similar to that of the same mixture under denaturing conditions (6 M Gdn-HCl, $\mathrm{pH} 3.5$ ), with a dominant emission peak at $520 \mathrm{~nm}$ (donor emission). On the other hand, the spectrum of the same mixture after renaturation is largely different: the $520 \mathrm{~nm}$ emission peak of the donor is decreased, whereas a $570 \mathrm{~nm}$ peak due to acceptor emission appears. The obtained emission intensity of the acceptor is significantly higher than that of controls containing only acceptor excited at $480 \mathrm{~nm}$ (donor excitation) (Fig. S1B. Red spectrum). The spectral changes upon reassembly indicate the nonradiative energy transfer (FRET) between Alexa-488 and Alexa-555 due to the proximity of the conjugate probes in the ferritin shells, and thus their co-assembly. An obstacle in this system is the quantification of the FRET signal, since to the emission peak of the acceptor contribute both the direct acceptor emission and the actual FRET emission signal. This is overcome by the (ratio) $)_{\mathrm{A}}$ parameter, which is proportional to FRET efficiency. More specifically, the value (ratio) $)_{\mathrm{A}}$ is obtained from the emission value of the acceptor because of FRET divided by the total emission of the acceptor exited directly (Fig. S1C), and thus, changes in (ratio) A $_{\mathrm{A}}$ serve as a proxy for conformational changes studies $(30,35,36)$. Calculated values of $(\text { ratio })_{\mathrm{A}}$, show that when donor and acceptor fluorophores are coassembled, a high efficiency inter-subunit energy transfer takes place (Fig. 3D. Blue column). Values of 0.7 in FRET efficiencies are obtained in the reassembled ferritin shells with 1:1 donor:acceptor, to be compared with 0.2-0.3 FRET efficiency in the unfolded and not co-assembled mixtures (Fig. 3D. Grey and black columns). After having shown that the FRET approach is working, we applied it to the formation of heteropolymeric ferritin shells made of $\mathrm{H}$ - and L-chains. We used the same procedure except that we used FTL-555 in place of FTH-555, and that the denaturation of the more stable FTL was done in $8 \mathrm{M} \mathrm{Gdn}-\mathrm{HCl} \mathrm{pH} \mathrm{3.5.} \mathrm{The} \mathrm{spectra} \mathrm{of} \mathrm{the} \mathrm{mixture} \mathrm{before} \mathrm{and} \mathrm{after} \mathrm{assembly}$ and the relative (ratio) ${ }_{\mathrm{A}}$ values, shown in Fig. 3, $C$ and $E$, are comparable to those obtained for the homopolymeric ferritin, indicating that H- and L-chains can coassemble with similar efficiency.

\subsection{Kinetics of ferritin self-assembly in homopolymers and heteropolymers.}

Once established the conditions to follow ferritin assembly by FRET, we used them to study the kinetics of self-assembly, which has been studied so far mainly by slow electrophoretic approaches (16). We started studying the kinetics of FTH homopolymers assembly by mixing equimolar mixtures of unfolded H-488:H-555 chains and monitoring the spectra immediately after dilution in the renaturation buffer. Emission spectra under $480 \mathrm{~nm}$ excitation of triplicate samples were recorded every $30 \mathrm{~s}$ during the first 3 hours and then every hour until a total of 24 hours and from them, $(\text { ratio })_{\mathrm{A}}$ was calculated. The time-course plots of the (ratio) ${ }_{\mathrm{A}}$ show an exponential increase in energy transfer in the first $30 \mathrm{~min}$ of reaction reaching a plateau at 2 hours, with a calculated $\mathrm{k}$ of $6.67 \cdot 10^{-5} \mathrm{~s}^{-1}$ and $t_{1 / 2}$ of $40 \mathrm{~min}$ (Fig. 4A. Blue line). The controls containing the mixtures of disassembled chains and of the native FTH-488 and FTH-555 homopolymers show negligible changes in (ratio) $)_{\mathrm{A}}$ during the $24 \mathrm{~h}$ monitoring (Fig. 4A. Grey and black plots). To verify that the spectra reflected ferritin assembly, sample aliquots were taken at different reaction times $(5,30,120$ and 1440 minutes) and analyzed in non-denaturing PAGE, showing that after 30 min full assembled ferritin shells were quantitatively formed. We then performed the same experiments by using the acceptor fluorophore 555 bound to the $\mathrm{L}$ ferritin to monitor the formation of $\mathrm{H} / \mathrm{L}$ heteropolymers. The kinetics obtained were similar to those of homopolymers, except that the reactions were slightly faster 
(Fig. 4, B and C. Red plot), with a calculated k of $8.14 \cdot 10^{-5} \mathrm{~s}^{-1}$ and $\mathrm{t}_{1 / 2}$ of $32 \mathrm{~min}$. These results reveal the human ferritin self-assembly process over time, showing that the assembly of $\mathrm{H} / \mathrm{L}$ heteropolymers is faster compared to that of $\mathrm{H} / \mathrm{H}$ homopolymers and that both structures complete their assembly in less than one hour.

\subsection{FRET to characterize the formation of isoferritin hybrids.}

Next, we analyzed how FRET efficiency is affected by modifying donor:acceptor ratios. Constant amounts of unfolded H-488 were added of increasing amounts of unfolded acceptor H-555 or L-555-chains, reassembled and the spectra collected after $48 \mathrm{~h}$. In the control unfolded mixtures, $(\text { ratio) })_{\mathrm{A}}$ values were about 0.3 at all donor/acceptor proportions (Fig. 5A and B. Grey columns) but raised when these mixtures were reassembled (Fig. $5 A$ and B. Blue and red columns). The (ratio) A $_{\mathrm{A}}$ values obtained in the reassembled ferritins were negatively related to the increase of acceptor subunits, in accordance with previously reported studies on multiple-acceptor systems where insufficient emitting donors yield an insufficient excitation of the acceptors via energy transfer $(37,38)$. The absence of this tendency in the corresponding disassembled mixtures confirmed the reassembly of the ferritins at the pre-incubated donor:acceptor ratio. Furthermore, of interest was that molecules with $\mathrm{H}$ - or L-acceptor behaved similarly, confirming once again that the two chains coassemble with similar efficiency.

However, this approach did not explain why H/L heteropolymers formation is preferred over the $\mathrm{H} / \mathrm{H}$ homopolymers. To address this question, we designed a system capable to compare the effect of $\mathrm{H}$ - and L-chains in the assembly of the H-chains. More specifically, we used donor-acceptor Hchain couples (H-488 and H-555) as sensors of H-subunit proximity and analyzed how various proportions of unlabeled $\mathrm{H}$ - and L-chains interfered with this proximity (Fig. 6A. Scheme). We first thought to co-assemble few couples of labeled $\mathrm{H}$-chains $(\mathrm{H}-488+\mathrm{H}-555)$ with various proportion of unlabeled $\mathrm{H}$-chains to yield $\mathrm{H} / \mathrm{H}$ homopolymeric ferritins (Table 1). The intervening unlabeled $\mathrm{H}-$ chains keep the donor and acceptor probes far apart, and by decreasing their proportion the FRET signal is predicted to increase linearly if the distribution of the labeled and unlabeled subunits is random. We co-assembled one couple of labeled $\mathrm{H}(1 \mathrm{H}-488+1 \mathrm{H}-555)$ with 22 unlabeled ones, then two couples of labeled H $(2 \mathrm{H}-488+2 \mathrm{H}-555)$ with 20 unlabeled ones, three copies $(3 \mathrm{H}-488+3 \mathrm{H}-555)$ with $18 \mathrm{H}$, four copies $(4 \mathrm{H}-488+4 \mathrm{H}-555)$ with $16 \mathrm{H}$, five copies $(5 \mathrm{H}-488+5 \mathrm{H}-555)$ with $14 \mathrm{H}$, and six copies $(6 \mathrm{H}-488+6 \mathrm{H}-555)$ with $12 \mathrm{H}$. After they reached equilibrium at $24 \mathrm{~h}$, triplicated samples were analyzed by fluorescence spectroscopy and FRET efficiency expressed as (ratio) A $_{\text {, was }}$ calculated. The results show that the FRET efficiency increased linearly with the number of fluorescent $\mathrm{H}$-subunits in the assembled $\mathrm{H} / \mathrm{H}$ homopolymeric shells (Fig. $6 B$ and $\mathrm{S} 3 A$ ). Since the energy transfer (ratio) $)_{\mathrm{A}}$ is considered an index of subunit proximity, this result indicates a random distribution of the fluorescent $\mathrm{H}$-chains not affected by the intervening unlabeled $\mathrm{H}$-chains, what in turn results in a linear increase on FRET-efficiency as the fluorescent H-chains crowd the structure. In consequence, the obtained FRET-efficiency in $\mathrm{H} / \mathrm{H}$ homopolymers is only dependent on the number of fluorophores present on the shell.

Then we performed the same experiment by co-assembling the donor-acceptor H-chain couples $(\mathrm{H}-488+\mathrm{H}-555)$ with unlabeled L-chains to yield $\mathrm{H} / \mathrm{L}$ heteropolymers. In this case the plot of $(\text { ratio })_{\mathrm{A}}$ was not linear but sigmoid, with negligible changes in FRET efficiency after the addition 4, 6 and $8 \mathrm{H}$-chains, and a large increase after $12 \mathrm{H}$-chains (Fig. $6 C$ and S3B). The low FRET efficiency values obtained for the addition of 4,6 and $8 \mathrm{H}$-chains are comparable to the basal of $\mathrm{H} / \mathrm{H}$ homopolymer (Fig. 6B) and indicates that the H-chain distribution in the presence of L-chains is not random, but it occupies preferential sites in the 24-mer heteropolymeric shell which tend to separate the FRET couple. The easiest interpretation is that the L-chains tend to displace the H-chains in the formation of the subunit dimers, indicating a preferred heterodimeric $(\mathrm{H} / \mathrm{L})$ over homodimeric $(\mathrm{H} / \mathrm{H})$ association during $\mathrm{H} / \mathrm{L}$ heteropolymer formation, as strong FRET-efficiencies would be expected if $\mathrm{H} / \mathrm{H}$ homodimers were preferred.

In an approach to verify this hypothesis we co-assembled the FRET H-couple with unlabeled human mitochondrial ferritin chains (FTMT) which physiologically never co-assemble with the cytosolic $\mathrm{H}$ - and L-chains. The (ratio) $\mathrm{A}$ plot vs unlabeled mitochondrial chains was linear as for the $\mathrm{H} / \mathrm{H}$ homopolymers, confirming that they do not displace the H-chains in dimer formation ( $\mathrm{S} 4 \mathrm{~A}$ and 
$B$ ). These results support our hypothesis as FTMT is considered as a H-like ferritin which shares a high degree of sequence homology with human H-chain ferritin (79\%) and bears also the ferroxidase activity $(39,40)$.

The results shown here indicate that in the formation of the H/L heteropolymers the presence of the L-chain push the H-chains far apart, and this may have a functional effect on ferritin shells, since the H-chains carry the ferroxidase activity, while the L-chains do not. To approach this, we studied the ferroxidase activity of the heteropolymers with the same H/L composition as those shown in Figure $6 C$. The reassembled ferritin preparations at pH 6.5 were added increments of $1000 \mathrm{Fe}$ (II) atoms and the initial the variation of the absorbance at $310 \mathrm{~nm}$ measured (13). We found that the ferroxidase activity increases with the H-chain content until it reaches a plateau at $8 \mathrm{H}$-chains/shell (Fig. $7 A$ and $B$ ) in agreement with Santambrogio et al, 1993 (13). Of interest was that the saturation was at $8 \mathrm{H}$ chains, which are the heteropolymers in which the $\mathrm{H}$-chains are the most far apart according to our FRET measurements (Fig. 6C). This suggests that the steric distribution of the ferroxidase activities, i.e. the H-chains, has a role in ferritin heteropolymers functionality.

\section{Discussion}

The use of ferritin to encapsulate several low molecular weight molecules of pharmacological or chemical interest has strongly increased in the recent period $(3,4,41)$. It is based on the observation that ferritin can be disassembled at low or high $\mathrm{pH}$ and then reassembled in the presence of the chemical of interest that will remain trapped inside the large cavity $(42,43,44)$. The system is robust and can be applied to ferritin of various origins, including the human and bacterial ones. However, little attention has been paired so far to the reassembly reaction, although it is an interesting and simple model for the study of protein self-assembly processes. Pioneering studies were done by the group of Jaenicke in 1987 studying the commercial horse spleen ferritin (16) and then by Santambrogio et al. in 1993 using the recombinant human H ferritin (13). Both concluded that the reaction goes to completion in less than an hour, and that the subunit dimer is the first essential step in the assembly $(16,32,17)$. More recently the self-assembly reaction of bacterial ferritin EcFtnA has been studied by Small Angle-X-ray scattering technique (9), that suggested the existence of intermediate oligomers during the reaction and that it was complete in minutes. All these approaches considered the assembly of one subunit type to form homopolymers, but one interesting property of ferritin is the capacity to form heteropolymers made of different proportions of two subunit types, namely the $\mathrm{H}$ - and L-chain for mammalian ferritins. Previous work on horse spleen ferritin showed no inter-chain FRET when simultaneously labeling with Alexa Fluor dyes at exposed Lysines (45). However, the small Lys-Lys distance at adjacent subunits $(<1.6 \mathrm{~nm})$ can give raise to self-quenching of the fluorescence via fluorophore-fluorophore interactions and thus limit the energy transfer (46).

Here we showed that the self-assembly reaction of human ferritin can be monitored by FRET technique using conjugate fluorophores bound to exposed residues of the folded subunits. Cys90 is convenient because is the only thiol exposed at the outer surface of the ferritin shell and is specific of the H-chain, and the Lys are convenient because are also exposed and near the 2-fold axis. Moreover, the proximity of Cys90 and Lys86/87 in the assembled shell is such $(2.7 \mathrm{~nm})$ that the FRET energy transfer provides an indication of the formation of subunit dimers carrying the conjugate fluorophores. We used the donor fluorophore AF488 attached to H-chain and the acceptor AF555 to either the H- or L-chain, what allowed us to produce ferritin $\mathrm{H} / \mathrm{H}$ homopolymers and $\mathrm{H} / \mathrm{L}$ heteropolymers containing donor and acceptor subunits. The obtained FRET efficiency in the reassembled ferritins agreed with the computed one for the formation of subunit dimers (Table S1), and the designed FRET-system was able to discern between the assembled and disassembled structures in both ferritin homopolymers and heteropolymers. This allowed to follow the kinetics of self-assembly on $\mathrm{H} / \mathrm{H}$ homopolymers and $\mathrm{H} / \mathrm{L}$ heteropolymers at a 1:1 ratio. The obtained kinetic profiles showed that both structures complete their assembly in less than one hour, but the $\mathrm{H} / \mathrm{L}$ heteropolymer assembly was faster than the $\mathrm{H} / \mathrm{H}$ homopolymers one. This supports the preferential formation of heteropolymers over the homopolymers. It should be noted that the kinetics we observed by FRET and confirmed by PAGE are slower that those measured by small-angle X-ray scattering of the bacterial ferritin (9). The difference may be attributed to the different ferritin types and that SAXRS follows the subunit aggregation and cannot distinguish a well-formed shell from an aggregate made of incompletely 
folded and poorly stable subunits. The FRET signal is distance-dependent and our results have demonstrated that it occurs only with fully assembled dimers.

Another important aspect we approached is about the formation of the heteropolymers, or how the $\mathrm{H}-$ and $\mathrm{L}$-chains preferentially associate during the assembly in $\mathrm{H} / \mathrm{L}$ heteropolymers. The mechanism of this reaction of assembly is unclear, and it would be of interest to verify if the subunit distribution in the 24-mer heteropolymeric structure is random, or it follows a specific pattern directed by some specific interactions. We firstly added different proportions of acceptor respect to the donor. The results obtained with this approach showed that the two chains coassemble with similar efficiency, and the (ratio) $)_{\mathrm{A}}$ values were proportional to the fluorophore content per shell. More informative was the approach in which we analyzed how the addition of non-labeled chains affected the FRET between couples of donor and acceptor H-chains at controlled amounts. This showed that the addition of the L-chain had a much stronger effect than that of the H-chain in reducing FRET signal, and therefore in keeping the H-chain couples separated. This strongly supports the preferred formation of heterodimers $(\mathrm{H} / \mathrm{L})$ over homodimers $(\mathrm{H} / \mathrm{H})$ in a heteropolymeric shell, as high FRETefficiencies would be expected if $\mathrm{H}$-chain dimers were preferred. This evidence was simulated in silico by using the crystallographic structures of the human $\mathrm{H}$ and human L ferritin (PDB ID: 2FHA and 2FG4, respectively). Molecular Dynamics (MD) simulations of the $\mathrm{H} / \mathrm{H}$ dimer (See Supplementary Material) showed strong interactions in the region involving Arg79, Ser6 and Gln7, close to the N-terminus of one chain, and residues Asp44 and Asp45 of the other (Fig. S5). The Nterminal tail is 4 residues longer in the H-chain than in the L-chain, and one could argue that it could hinder the creation of some of the key contacts that eventually lead to the assembly of the dimer. In other terms, a higher loss of entropy is associated to the $\mathrm{H} / \mathrm{H}$ dimer formation with respect to the $\mathrm{H} / \mathrm{L}$ one. This hypothesis should be supported by further investigation, based on accurate calculations of the binding free energies of dimers. Such an analysis is beyond the scope of the present work.

The FRET patterns of the heteropolymers indicated that the maximum interference of the Lchains on the H-chains coming together occurred in the $8 \mathrm{H} / 16 \mathrm{~L}$ hybrid, where the $\mathrm{H}$-chains were showed to be placed at distant positions (Fig. 6C). If we assume that in these shells the $\mathrm{H}$ are as far apart as possible, then each of them should occupy a position at each of the eight 3 -fold channels. Interestingly the kinetic experiments of Figure 7 show that these shells are the ones with the highest capacity of iron oxidation and that a further increase in H-chain content did not increase the rate of iron oxidation/incorporation. This observation suggests that participation of a single $\mathrm{H}$-chain at 3-fold channel is sufficient for the highest ferritin activity, at least under the conditions of high iron increments $(1,000 \mathrm{Fe} / \mathrm{shell})$, despite that the residues exposed to the channel are conserved in $\mathrm{H}$ and $\mathrm{L}$ chains. This opens the way to various interpretations, one of which is that the proximity of a single ferroxidase center to the channel is sufficient to facilitate iron entry the cavity for its oxidation, and the presence of a second or third one would not improve it. Alternatively, the preferred formation of the subunit heterodimers may facilitate the activity of iron oxidation/incorporation by putting the two major functions of iron oxidation of the H-chain and of iron nucleation of the L-chain in close proximity. Altogether the data indicate that the distribution of the ferroxidase activity, i.e. the Hchains, in the ferritin shells have an impact on their functionality. The in vitro re-assembly may reflect the in vivo formation of the isoferritins. If this is the case, then in the liver or in condition of tissue iron overload when the L-chain predominates and the $\mathrm{H}$ proportion is $<8 / 24$, the specific ferritin functionality is reduced, but maybe this is compensated by the abundance of total ferritins. Moreover, we observed that when the $\mathrm{H}$ subunits were assembled together with the "H-like" FTMT, which holds $70 \%$ identity with $\mathrm{H}$-chain and also has ferroxidase activity, the assembly behaves like a $\mathrm{H} / \mathrm{H}$ homopolymeric ferritin and the patterned arrangement of the $\mathrm{H}$ subunits disappears.

Conflict of interest: The authors declare that they have no conflicts of interest with the contents of this article.

Author contributions: FC conceived, performed and analyzed all the experiments and wrote most of the paper. MP, designed and constructed vectors for expression of recombinant ferritins. MB and AG performed the MALDI-TOF analyses shown in Suppl. $S 2$ and Table $S 2$. FG performed the in-silico 
study shown in Suppl. S5. PA conceived the idea for the project and wrote the paper with FC. All authors analyzed the results and approved the final version of the manuscript.

Funding: This work was partially supported by MIUR grant PRIN10-11 to PA, and by Telethon grant GGP15064 to PA. FC was recipient of a Post-Doc Fellowship from University of Brescia, and was partially supported by CIB (Consorzio Italiano di Biotecnologie).

\section{References}

1. Harrison, P. M., and Arosio, P. (1996) The ferritins: molecular properties, iron storage function and cellular regulation. BBA-Bioenergetics. 1275, 161-203.

2. Arosio, P., Carmona, F., Gozzelino, R., Maccarinelli, F., and Poli, M. (2015) The importance of eukaryotic ferritins in iron handling and cytoprotection. Biochem. J. 472, 1-15.

3. Jutz, G., Van Rijn, P., Santos Miranda, B., and Böker, A. (2015) Ferritin: a versatile building block for bionanotechnology. Chem. Rev. 115, 1653-1701.

4. He, D., and Marles-Wright, J. (2015) Ferritin family proteins and their use in bionanotechnology. New biotechnol. 32, 651-657.

5. Lin, X., Xie, J., Niu, G., Zhang, F., Gao, H., Yang, M., and Leapman, R. (2011) Chimeric ferritin nanocages for multiple function loading and multimodal imaging. Nano lett. 11, 814819.

6. Stefanini, S., Vecchini, P., and Chiancone, E. (1987) On the mechanism of horse spleen apoferritin assembly: a sedimentation velocity and circular dichroism study. Biochemistry 26, 1831-1837.

7. Zhang, Y., and Orner, B. P. (2011) Self-assembly in the ferritin nano-cage protein superfamily. Int. J. Mol. Sci. 12, 5406-5421.

8. Chen, H., Zhang, S., Xu, C., and Zhao, G. (2016) Engineering protein interfaces yields ferritin disassembly and reassembly under benign experimental conditions. Chem. Comm. 52, 74027405 .

9. Sato, D., Ohtomo, H., Yamada, Y., Hikima, T., Kurobe, A., Fujiwara, K., and Ikeguchi, M. (2016) Ferritin Assembly Revisited: A Time-Resolved Small-Angle X-ray Scattering Study. Biochemistry 55, 287-293.

10. Corsi, B., Perrone, F., Bourgeois, M., Beaumont, C., Panzeri, C. M., Cozzi, A., Sangregorio, R., Santambrogio, P., Albertini, P., Arosio, P., and Levi, S. (1998) Transient overexpression of human $\mathrm{H}$-and L-ferritin chains in COS cells. Biochem. J. 330, 315-320.

11. Cozzi, A., Corsi, B., Levi, S., Santambrogio, P., Albertini, A., and Arosio, P. (2000) Overexpression of wild type and mutated human ferritin H-chain in HeLa cells in vivo role of ferritin ferroxidase activity. J. Biol. Chem. 275, 25122-25129.

12. Luscieti, S., Santambrogio, P., d'Estaintot, B. L., Granier, T., Cozzi, A., Poli, M., Gallois, B., Finazzi, D., Cattaneo, A., Levi, S., and Arosio, P. (2010) Mutant ferritin L-chains that cause neurodegeneration act in a dominant-negative manner to reduce ferritin iron incorporation. $J$. Biol. Chem. 285, 11948-11957.

13. Santambrogio, P., Levi, S., Cozzi, A., Rovida, E., Albertini, A., and Arosio, P. (1993) Production and characterization of recombinant heteropolymers of human ferritin $\mathrm{H}$ and $\mathrm{L}$ chains. J. Biol. Chem. 268, 12744-12748.

14. Kim, M., Rho, Y., Jin, K. S., Ahn, B., Jung, S., Kim, H., and Ree, M. (2011) pH-dependent structures of ferritin and apoferritin in solution: disassembly and reassembly. Biomacromolecules 12, 1629-1640.

15. Santambrogio, P., Levi, S., Arosio, P., Palagi, L., Vecchio, G., Lawson, D. M., Yewda, S., Artymiuk, P., Harrison, P. M., and Jappelli, R. (1992) Evidence that a salt bridge in the light chain contributes to the physical stability difference between heavy and light human ferritins. J. Biol. Chem. 267, 14077-14083.

16. Gerl, M., and Jaenicke, R. (1987) Mechanism of the self-assembly of apoferritin from horse spleen. Eur. Biophys. J. 15, 103-109.

17. Santambrogio, P., Pinto, P., Levi, S., Cozzi, A., Rovida, E., Albertini, A., Artymiuk, P., 
Harrison, P. M., Jappelli, R., and Arosio, P. (1997) Effects of modifications near the 2-, 3-and 4-fold symmetry axes on human ferritin renaturation. Biochem. J. 322, 461-468.

18. Huard, D. J., Kane, K. M., and Tezcan, F. A. (2013) Re-engineering protein interfaces yields copper-inducible ferritin cage assembly. Nat. Chem. Biol. 9, 169-176.

19. Rucker, P., Torti, F. M., Torti, S. V. (1997) Recombinant ferritin: modulation of subunitstoichiometry in bacterial expression systems. Protein Eng. 10, 967-73.

20. Miyawaki, A., and Tsien, R. Y. (2000) Monitoring protein conformations and interactions by fluorescence resonance energy transfer between mutants of green fluorescent protein. Method. Enzymol. 327, 472-500.

21. Sekar, R. B., and Periasamy, A. (2003) Fluorescence resonance energy transfer (FRET) microscopy imaging of live cell protein localizations. J. Cell Biol. 160, 629-633.

22. Carmona, F., Muñoz-Robles, V., Cuesta, R., Gálvez, N., Capdevila, M., Maréchal, J. D., and Dominguez-Vera, J. M. (2014) Monitoring lactoferrin iron levels by fluorescence resonance energy transfer: a combined chemical and computational study. J. Biol. Inorg. Chem. 19, 439447.

23. Hong, S. H., and Maret, W. (2003) A fluorescence resonance energy transfer sensor for the $\beta$ domain of metallothionein. P. Natl. A. Sci. 100, 2255-2260.

24. Lindhoud, S., Westphal, A. H., van Mierlo, C. P., Visser, A. J., and Borst, J. W. (2014) Risetime of FRET-acceptor fluorescence tracks protein folding. Int. J. Mol. Sci. 15, 23836-23850.

25. Santambrogio, P., Cozzi, A., Levi, S., Rovida, E., Magni, F., Albertini, A., and Arosio, P. (2000) Functional and immunological analysis of recombinant mouse H- and L-ferritins from Escherichia coli. Prot. Exp. Purif. 19, 212-218.

26. Gianoncelli, A., Bonini, S.A., Bertuzzi, M., Guarienti, M., Vezzoli, S., Kumar, R., Delbarba, A., Mastinu, A., Sigala, S., Spano, P., Pani, L., Pecorelly, S., and Memo, M. (2015) An integrated approach for a structural and functional evaluation of biosimilars: implications for erythropoietin. BioDrugs 29, 285-300.

27. Lakowicz, J.R. (2006) Principles of Fluorescence Spectroscopy 3rd Ed., Plenum 443 p. New York.

28. Martin, S. F., Tatham, M. H., Hay, R. T., and Samuel, I. D. (2008) Quantitative analysis of multi-protein interactions using FRET: Application to the SUMO pathway. Prot. Sci. 17, 777 784.

29. Chakraborty, S., Núñez, D., Hu, S. Y., Domingo, M. P., Pardo, J., Karmenyan, A., Gálvez, E. M., and Chiou, A. (2014) FRET based quantification and screening technology platform for the interactions of leukocyte function-associated antigen-1 (LFA-1) with intercellular adhesion molecule-1 (ICAM-1). PloS one 9, e102572.

30. Smiley, R. D., Collins, T. R., Hammes, G. G., and Hsieh, T. S. (2007) Single-molecule measurements of the opening and closing of the DNA gate by eukaryotic topoisomerase II. $P$. Natl. A. Sci. 104, 4840-4845

31. Booth, E. A., Vane, E. W., Dovala, D., and Thorner, J. (2015) A Förster resonance energy transfer (FRET)-based system provides insight into the ordered assembly of yeast septin hetero-octamers. J. Biol. Chem. 290, 28388-28401.

32. Gerl, M., Jaenicke, R., Smith, J. M., and Harrison, P. M. (1988) Self-assembly of apoferritin from horse spleen after reversible chemical modification with 2,3-dimethylmaleic anhydride. Biochemistry 27, 4089-4096.

33. Levi, S., Luzzago, A., Franceschinelli, F., Santambrogio, P., Cesareni, G., and Arosio, P. (1989) Mutational analysis of the channel and loop sequences of human ferritin H-chain. Biochem. J. 264, 381-388.

34. Panchuk-Voloshina, N., Haugland, R. P., Bishop-Stewart, J., Bhalgat, M. K., Millard, P. J., Mao, F., Leung, W. Y., and Haugland, R. P. (1999) Alexa dyes, a series of new fluorescent dyes that yield exceptionally bright, photostable conjugates. J. Histochem. Cytochem. 47, 1179-1188.

35. Sternberg, S. H., LaFrance, B., Kaplan, M., and Doudna, J. A. (2015) Conformational control of DNA target cleavage by CRISPR-Cas9. Nature 527, 110-113.

36. Majumdar, Z. K., Hickerson, R., Noller, H. F., and Clegg, R. M. (2005) Measurements of 
internal distance changes of the $30 \mathrm{~S}$ ribosome using FRET with multiple donor-acceptor pairs: quantitative spectroscopic methods. J. Mol. Biol. 351, 1123-1145.

37. Lichlyter, D. J., Grant, S. A., and Soykan, O. (2003) Development of a novel FRET immunosensor technique. Biosens. Bioelectron. 19, 219-226.

38. Bojarski, P., Kulak, L., Walczewska-Szewc, K., Synak, A., Marzullo, V. M., Luini, A., and D’Auria, S. (2011) Long-distance FRET analysis: A Monte Carlo simulation study. J. Phys. Chem. B. 115, 10120-10125.

39. Levi, S., Corsi, B., Bosisio, M., Invernizzi, R., Volz, A., Sanford, D., Arosio, P., and Drysdale, J. (2001) A human mitochondrial ferritin encoded by an intronless gene. J. Biol. Chem. 276, 24437-24440.

40. Bou-Abdallah, F., Santambrogio, P., Levi, S., Arosio, P., and Chasteen, N. D. (2005) Unique iron binding and oxidation properties of human mitochondrial ferritin: a comparative analysis with human H-chain ferritin. J. Mol. Biol. 347, 543-554).

41. Uchida, M., Kang, S., Reichhardt, C., Harlen, K., and Douglas, T. (2010) The ferritin superfamily: Supramolecular templates for materials synthesis. BBA-Gen. Subjects. 1800, 834-845.

42. Yang, Z., Wang, X., Diao, H., Zhang, J., Li, H., Sun, H., and Guo, Z. (2007) Encapsulation of platinum anticancer drugs by apoferritin. Chem. Comm. 33, 3453-3455.

43. Domínguez-Vera, J. M., and Colacio, E. (2003) Nanoparticles of Prussian blue ferritin: a new route for obtaining nanomaterials. Inorg. Chem. 42, 6983-6985.

44. Chen, L., Bai, G., Yang, S., Yang, R., Zhao, G., Xu, C., and Leung, W. (2014) Encapsulation of curcumin in recombinant human $\mathrm{H}$-chain ferritin increases its water-solubility and stability. Food Res. Int. 62, 1147-1153.

45. Fernández, B., Gálvez, N., Sánchez, P., Cuesta, R., Bermejo, R., and Domínguez-Vera, J. M. (2008) Fluorescence resonance energy transfer in ferritin labeled with multiple fluorescent dyes. J. Biol. Inorg. Chem. 13(3), 349-355.

46. Ogawa, M., Kosaka, N., Choyke, P. L., and Kobayashi, H. (2009) H-type dimer formation of fluorophores: a mechanism for activatable, in vivo optical molecular imaging. ACS Chemical Biology 4(7), 535-546. 


\section{FIGURE LEGENDS}

TABLE 1. Composition of the produced $\mathrm{H} / \mathrm{L}$ heteropolymers and $\mathrm{H} / \mathrm{H}$ homopolymers containing different labeled and unlabeled subunit proportions. H-488, H-chain labeled with AF488; H-555, Hchain labeled with AF555.

FIGURE 1. Ferritin structure and measured inter-chain distances. A, 24-meric structure of FTH. Bottom: Ferritin H- and L-chains (PDB ID: 2FHA, 2FG4, respectively) showing exposed side chains of targeted lysines (yellow sticks) and cysteine (red sticks); $B$, representation of the environment surrounding each subunit in a 24-mer shell. One subunit (cyan) can interact with other six subunits (blue) through noncovalent interactions. Inter-subunit distances, from Cys90 (red) on reference subunit to Lys86/87 (yellow) of the surrounding ones, are shown. Inset, schematic view of the FRET system at the 2-fold axes, where inter-subunit distances are reported to be the smallest, with fluorophore AF488 attached to Cys90 represented as green star and AF555 attached to Lys86 as magenta star.

FIGURE 2. Labeling of $\mathrm{H}$ and $\mathrm{L}$ ferritins for FRET analysis. $A$, gel filtration elution profiles of the fluorescently-labeled ferritins. Protein elution was monitored by its absorbance at $280 \mathrm{~nm}$ (black line) while AF488 or AF555 were monitored at 488 and $555 \mathrm{~nm}$ respectively (green and pink lines). Coelution of the fluorophore and the protein indicates that they are attached. $B, 7 \%$ non-denaturing PAGE of the purified fluorescent homopolymers showing images of non-stained, UV-scanned and finally Coomassie blue stained gel, confirming the covalent binding of the fluorophores to the proteins. The slow moving labeled species represent the ferritin shell dimers, which are always present in the ferritin preparation. $C$, Representation of the fluorescent ferritins synthesized and normalized excitation and emission spectra of FTH-488 and FTH-555. Extensive spectral overlap occurs between the emission of Alexa Fluor 488 and excitation of Alexa Fluor 555, being thus a suitable couple for FRET studies.

FIGURE 3. FRET-based detection of ferritin assembly. $A$, schematic illustration of the reaction carried out for these FRET experiments. Donor FTH-488 is represented as green structures and acceptor FTH-555 as pink ones. When there is no coassembly, excitation of donor at $480 \mathrm{~nm}$ leads to only donor emission peak at $520 \mathrm{~nm}$. When coassembled, excitation at $480 \mathrm{~nm}$ leads to acceptor emission at $570 \mathrm{~nm}$, due to FRET. B, emission spectra upon donor excitation $(480 \mathrm{~nm})$, of reassembled full-labeled homopolymers (FTH-488/555, blue graph), disassembled chains $(\mathrm{H}-488+\mathrm{H}-$ 555, grey spectra) and not coassembled full-labeled homopolymers (FTH-488+FTH-555, black graph), evidencing the quenching at the donor emission around $520 \mathrm{~nm}$, together with an increase of the acceptor emission at $570 \mathrm{~nm}$ only when coassembled. $C$, emission spectra upon donor excitation $(480 \mathrm{~nm})$, of reassembled full-labeled heteropolymers (FTHL-488/555, red graph), disassembled chains (H-488+L-555, grey spectra) and not coassembled full-labeled shells (FTH-488+FTL-555, black graph), evidencing the quenching at the donor emission around $520 \mathrm{~nm}$, together with an increase of the acceptor emission at $570 \mathrm{~nm}$ when coassembled. $D$ and $E$, calculated (ratio) A values from the above emission spectra, evidencing the pre-requisite of coassembly for having FRET as

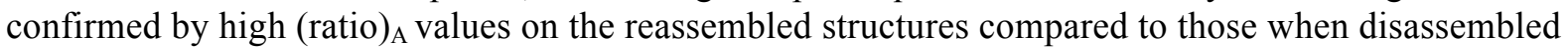
or not coassembled. Results are represented as means \pm SD of triplicate experiments.

FIGURE 4. Kinetics of ferritin self-assembly in homopolymers and heteropolymers. $A$ and $B$, kinetics of $\mathrm{H} / \mathrm{H}$ homopolymeric (blue) and $\mathrm{H} / \mathrm{L}$ heteropolymeric (red) assembly depicted by (ratio) ${ }_{\mathrm{A}}$. An exponential increase on FRET-efficiency is observed for both assemblies until they reach a plateau after 2 hours. Rate constant and half-time calculations reveal a faster assembly for the heteropolymers. Controls containing disassembled chains (grey) and not coassembled ferritins (black) were also monitored showing negligible changes in (ratio) $)_{\mathrm{A}}$ during time. Aliquots were taken at different times of assembly (5, 30, 120 and 1440 minutes) and analyzed in non-denaturing gel electrophoresis, confirming that after 30 minutes full assembled shells are already formed for both ferritin structures. $C$, comparison of the kinetics at the first 30 minutes for heteropolymer (red) and 
homopolymer (blue) evidencing the faster time-course of the heteropolymeric assembly. Results are represented as means $\pm \mathrm{SD}$ of triplicate experiments.

FIGURE 5. FRET properties of isoferritins containing differents donor:acceptor ratios. $A$ and $B$, $(\text { ratio })_{\mathrm{A}}$ values of reassembled and disassembled structures of isoferritins containing increasing acceptor ratios. Homopolymeric (blue) and heteropolymeric (red) structures seem to co-assemble with similar efficiency and show a (ratio) A $_{\text {p }}$ pattern that is a fingerprint of the content of acceptor subunits present on the shell and that is absent on the homolog disassembled mixtures. Lower Part, nondenaturing PAGE of the reassembled full-labeled ferritins containing different donor:acceptor ratios, scanned under UV light and Coomassie stained, which shows a protein ladder in agreement with their subunit composition. Results are represented as means \pm SD of triplicate experiments.

FIGURE 6. Design of a system capable to compare the effect of H- and L-chains in the assembly of the H-chain. A, scheme of reaction. A system capable to compare the effect of $\mathrm{H}$ - and L-chains in the assembly of the H-chains was designed. More specifically, we used donor-acceptor H-chain couples (H-488 and H-555, green and magenta structures) as sensors of $\mathrm{H}$-subunit proximity and analyzed how various proportions of unlabeled H- and L-chains (grey structures) interfered with this proximity. An association of the FRET $H$ subunits in the final shell would yield high FRET-efficiencies while low FRET would be expected if placed at distant positions. $B$, plotting of the (ratio) $)_{\mathrm{A}}$ values obtained for the assembly of different amounts of FRET couples $(\mathrm{H}-488+\mathrm{H}-555)$ together with unlabeled Hchains. The FRET-efficiency increases linearly with the number of fluorescent $\mathrm{H}$-subunits indicating a random distribution of the fluorescent $\mathrm{H}$-chains not affected by the intervening unlabeled H-chains. Bottom, plotting of the increments in (ratio) $)_{A}$ with respect to the number of fluorescent subunits per shell. $C$, plotting of the (ratio) $)_{\mathrm{A}}$ values obtained for the assembly of different amounts of FRET couples $(\mathrm{H}-488+\mathrm{H}-555)$ together with unlabeled L-chains. Negligible changes in FRET after the addition of 20, 18 and 16 L-chains, and a large increase after 12 L-chains, indicating that the H-chain distribution in the presence of L-chains is not random, but it occupies preferential sites in the 24-mer heteropolymeric shell which tend to separate the FRET couple. Bottom, plotting of the increments in $(\text { ratio })_{\mathrm{A}}$ with respect to the number of fluorescent subunits per shell highlighting the strong jump on $(\text { ratio })_{\mathrm{A}}$ when more than $8 \mathrm{H}$-chains/molecule. Results are represented as means $\pm \mathrm{SD}$ of triplicate experiments.

FIGURE 7. $A$. Progression plots of iron uptake monitored by absorbance at $310 \mathrm{~nm}$ of H/L heteropolymers containing a total of 4, 6, 8, 10 and 12 H-chains. Homopolymeric FTH (24 H-chains) and control containing Fe(II) in the absence of ferritins are also showed. $B$. Initial rates of iron uptake of the ferritins showed in $A$. Conditions: $0.1 \mu \mathrm{M}$ apoferritins, $0.1 \mathrm{M}$ Tris-HCL pH 6.5, $0.1 \mathrm{mM}$ ferrous ammonium sulfate, $25^{\circ} \mathrm{C}$. Results of triplicate experiments are represented as means $\pm \mathrm{SD}$. 


\section{TABLE 1}

\begin{tabular}{ccccc}
\hline $\begin{array}{c}\text { Ferritin } \\
\text { shell type }\end{array}$ & $\begin{array}{c}\text { (H-488+H-555) } \\
\text { chains }\end{array}$ & $\begin{array}{c}\text { Unlabeled } \\
\text { L-chains }\end{array}$ & $\begin{array}{c}\text { Unlabeled } \\
\text { H-chains }\end{array}$ & $\begin{array}{c}\text { (H-488+H-555) } \\
\text { to unlabeled } \\
\text { ratio }\end{array}$ \\
\hline & 4 & 20 & - & $1: 5$ \\
HETEROPOLYMER & 6 & 18 & - & $1: 3$ \\
(H/L) & 8 & 16 & - & $1: 2$ \\
& 10 & 14 & - & $1: 1.4$ \\
\hline \multirow{2}{*}{ HOMOPOLYMER } & 12 & 12 & - & $1: 1$ \\
(H/H) & 4 & - & 20 & $1: 5$ \\
& 8 & - & 18 & $1: 3$ \\
& 10 & - & 16 & $1: 2$ \\
& 12 & - & 14 & $1: 1.4$ \\
\end{tabular}

TABLE 1. Composition of the produced $H / L$ heteropolymers and $H / H$ homopolymers containing different labeled and unlabeled subunit proportions. H-488, H-chain labeled with AF488; H-555, Hchain labeled with AF555. 
FIG 1

A
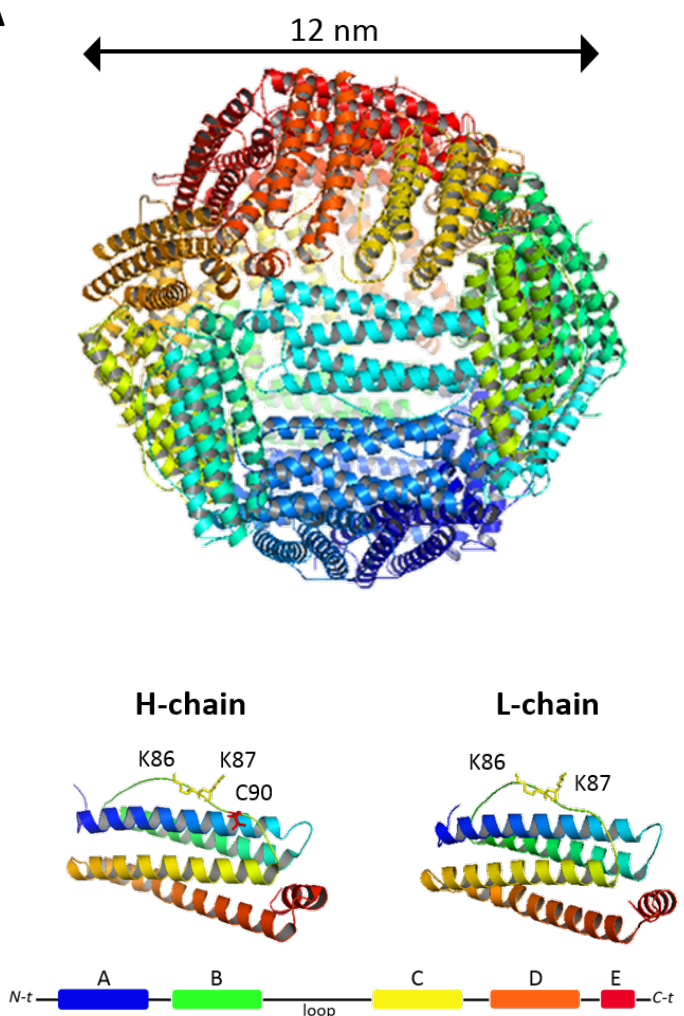

B

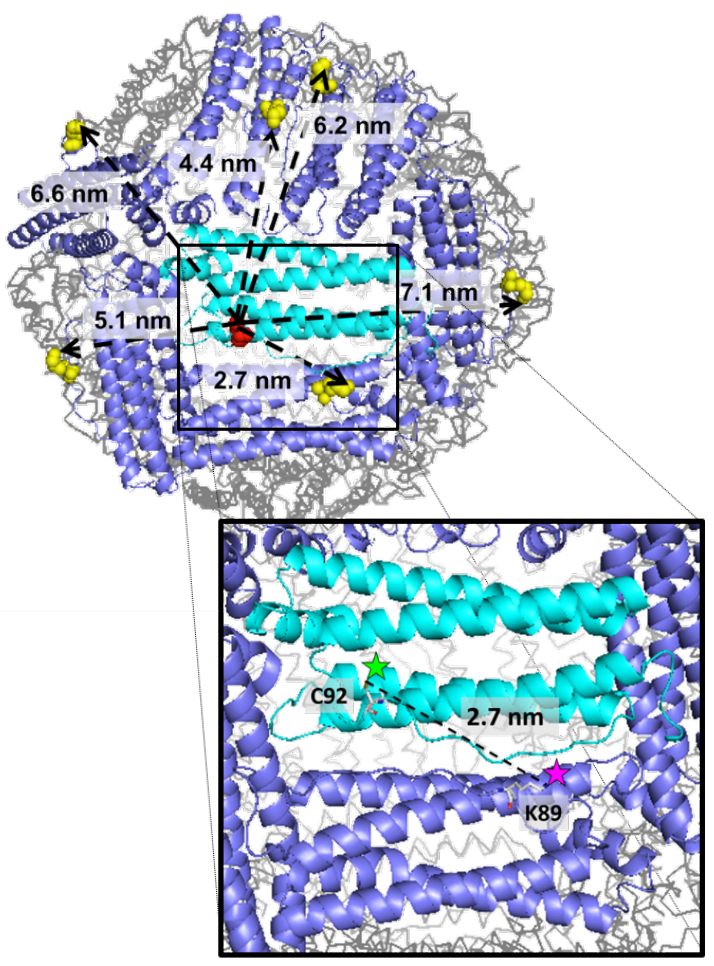

FIGURE 1. Ferritin structure and measured inter-chain distances. A, 24-meric structure of FTH. Bottom: Ferritin H- and L-chains (PDB ID: 2FHA, 2FG4, respectively) showing exposed side chains of targeted lysines (yellow sticks) and cysteine (red sticks); $B$, representation of the environment surrounding each subunit in a 24-mer shell. One subunit (cyan) can interact with other six subunits (blue) through noncovalent interactions. Inter-subunit distances, from Cys90 (red) on reference subunit to Lys86/87 (yellow) of the surrounding ones, are shown. Inset, schematic view of the FRET system at the 2-fold axes, where inter-subunit distances are reported to be the smallest, with fluorophore AF488 attached to Cys90 represented as green star and AF555 attached to Lys86 as magenta star. 
FIG 2

A

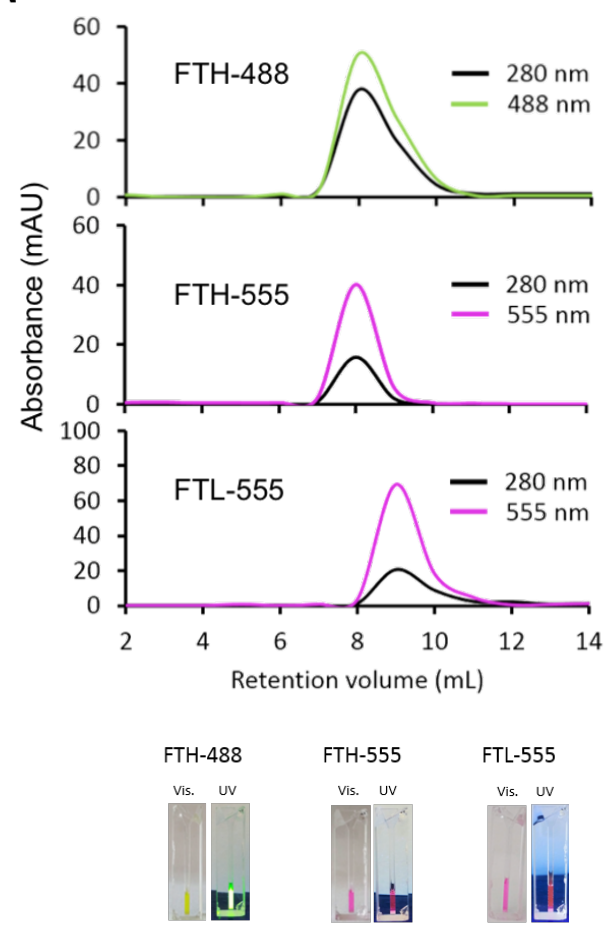

B

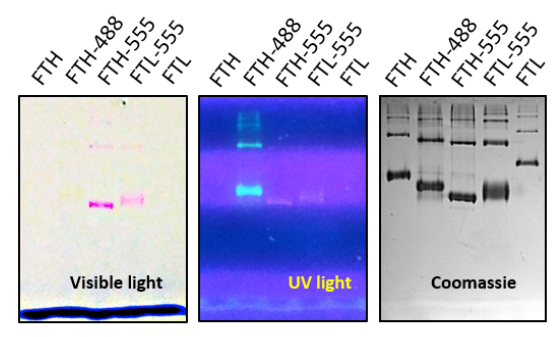

C
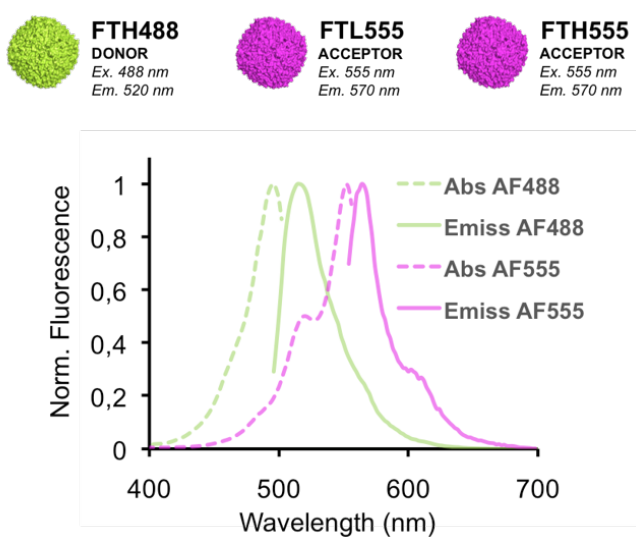

FIGURE 2. Labeling of $\mathrm{H}$ and $\mathrm{L}$ ferritins for FRET analysis. $A$, gel filtration elution profiles of the fluorescently-labeled ferritins. Protein elution was monitored by its absorbance at $280 \mathrm{~nm}$ (black line) while AF488 or AF555 were monitored at 488 and $555 \mathrm{~nm}$ respectively (green and pink lines). Coelution of the fluorophore and the protein indicates that they are attached. $B, 7 \%$ non-denaturing PAGE of the purified fluorescent homopolymers showing images of non-stained, UV-scanned and finally Coomassie blue stained gel, confirming the covalent binding of the fluorophores to the proteins. The slow moving labeled species represent the ferritin shell dimers, which are always present in the ferritin preparation. $C$, Representation of the fluorescent ferritins synthesized and normalized excitation and emission spectra of FTH-488 and FTH-555. Extensive spectral overlap occurs between the emission of Alexa Fluor 488 and excitation of Alexa Fluor 555, being thus a suitable couple for FRET studies. 
FIG 3

A

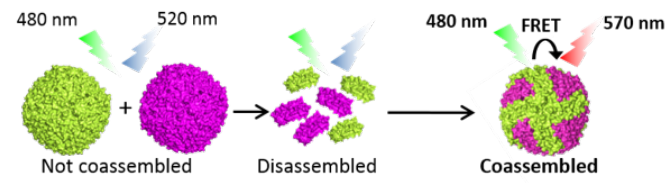

B

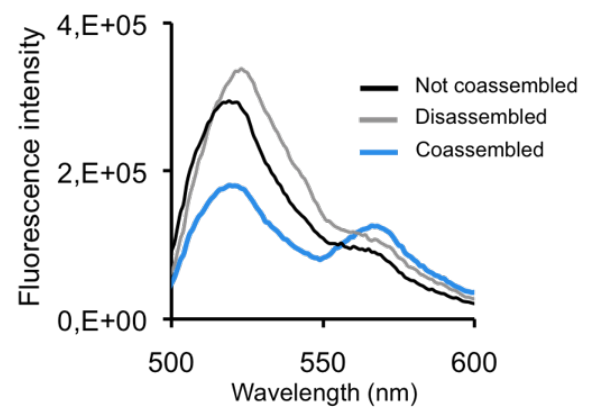

D

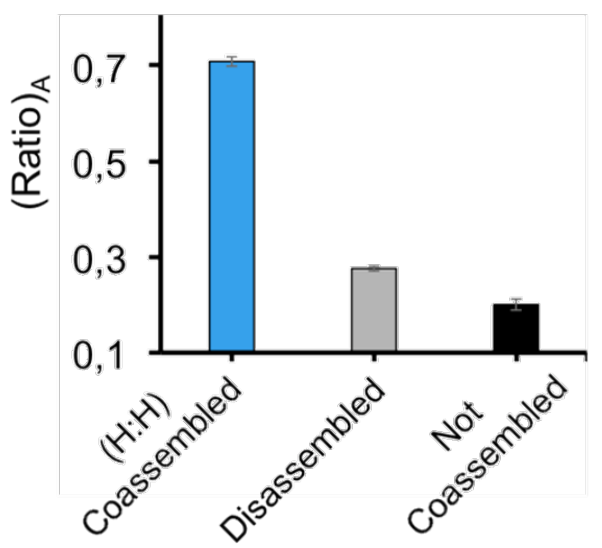

C

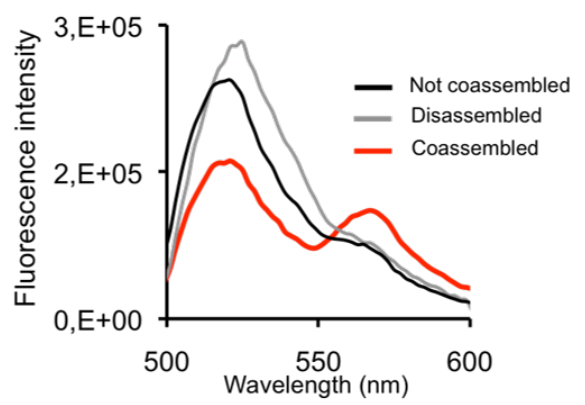

$\mathbf{E}$

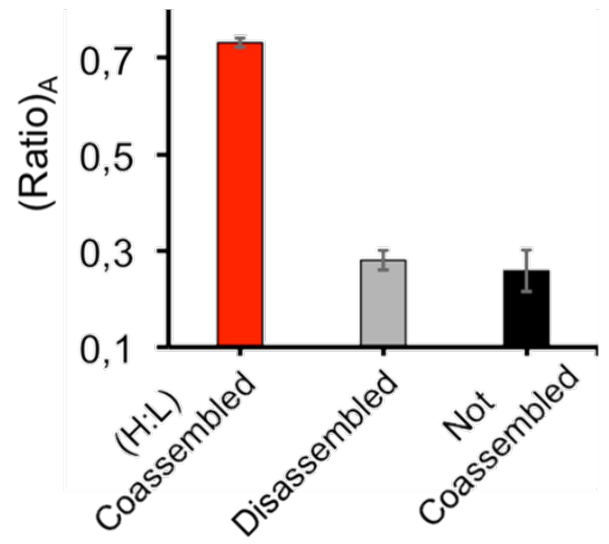

FIGURE 3. FRET-based detection of ferritin assembly. $A$, schematic illustration of the reaction carried out for these FRET experiments. Donor FTH-488 is represented as green structures and acceptor FTH-555 as pink ones. When there is no coassembly, excitation of donor at $480 \mathrm{~nm}$ leads to only donor emission peak at $520 \mathrm{~nm}$. When coassembled, excitation at $480 \mathrm{~nm}$ leads to acceptor emission at $570 \mathrm{~nm}$, due to FRET. B, emission spectra upon donor excitation $(480 \mathrm{~nm})$, of reassembled full-labeled homopolymers (FTH-488/555, blue graph), disassembled chains $(\mathrm{H}-488+\mathrm{H}-$ 555, grey spectra) and not coassembled full-labeled homopolymers (FTH-488+FTH-555, black graph), evidencing the quenching at the donor emission around $520 \mathrm{~nm}$, together with an increase of the acceptor emission at $570 \mathrm{~nm}$ only when coassembled. $C$, emission spectra upon donor excitation $(480 \mathrm{~nm})$, of reassembled full-labeled heteropolymers (FTHL-488/555, red graph), disassembled chains (H-488+L-555, grey spectra) and not coassembled full-labeled shells (FTH-488+FTL-555, black graph), evidencing the quenching at the donor emission around $520 \mathrm{~nm}$, together with an increase of the acceptor emission at $570 \mathrm{~nm}$ when coassembled. $D$ and $E$, calculated (ratio) A values from the above emission spectra, evidencing the pre-requisite of coassembly for having FRET as

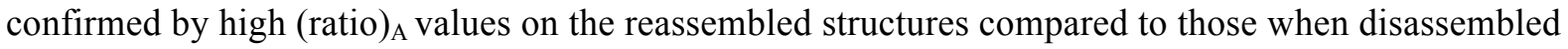
or not coassembled. Results are represented as means \pm SD of triplicate experiments. 
FIG 4
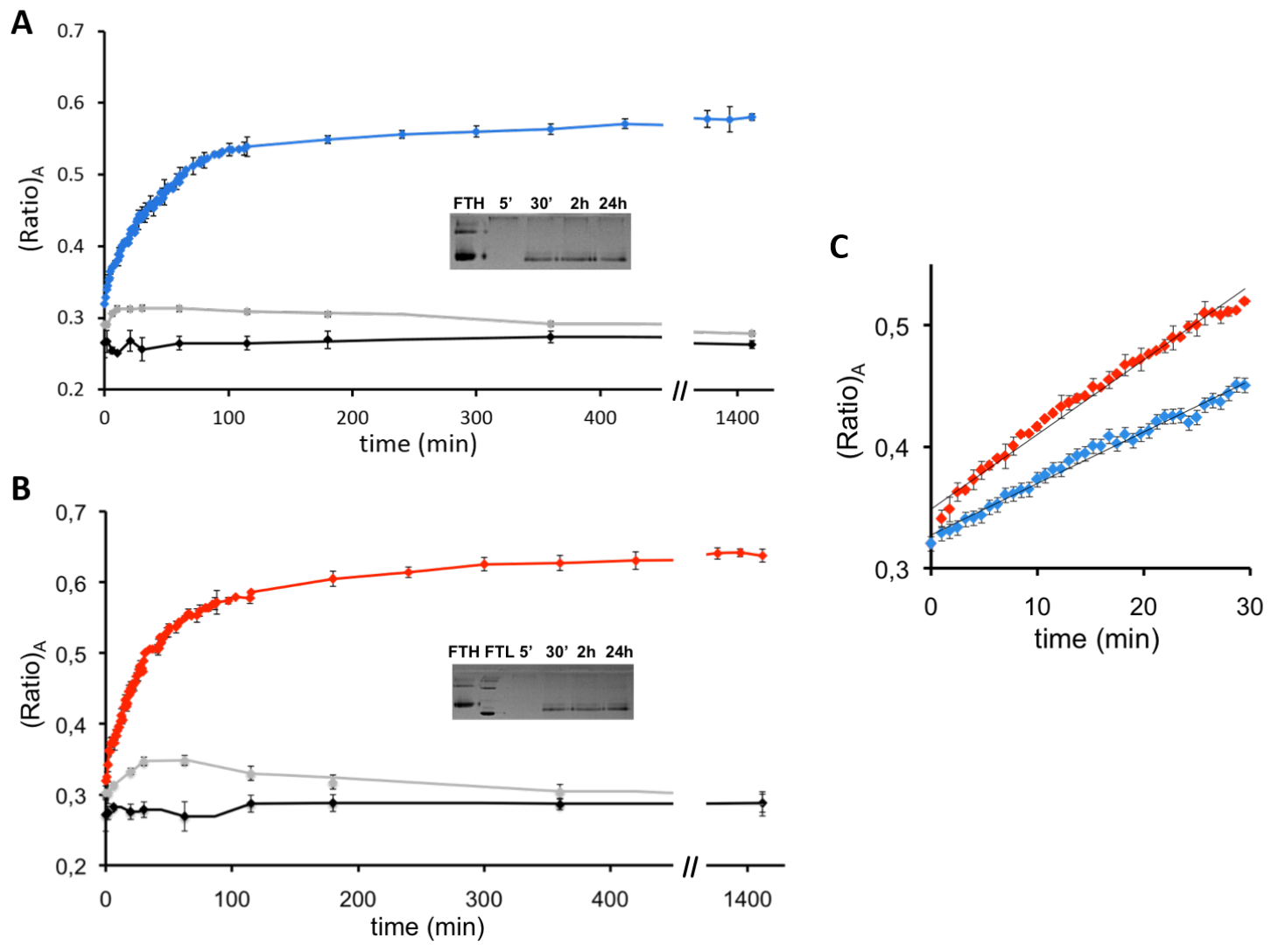

FIGURE 4. Kinetics of ferritin self-assembly in homopolymers and heteropolymers. $A$ and $B$, kinetics of $\mathrm{H} / \mathrm{H}$ homopolymeric (blue) and $\mathrm{H} / \mathrm{L}$ heteropolymeric (red) assembly depicted by (ratio) A $_{\mathrm{A}}$. An exponential increase on FRET-efficiency is observed for both assemblies until they reach a plateau after 2 hours. Rate constant and half-time calculations reveal a faster assembly for the heteropolymers. Controls containing disassembled chains (grey) and not coassembled ferritins (black) were also monitored showing negligible changes in (ratio) $)_{\mathrm{A}}$ during time. Aliquots were taken at different times of assembly (5, 30, 120 and 1440 minutes) and analyzed in non-denaturing gel electrophoresis, confirming that after 30 minutes full assembled shells are already formed for both ferritin structures. $C$, comparison of the kinetics at the first 30 minutes for heteropolymer (red) and homopolymer (blue) evidencing the faster time-course of the heteropolymeric assembly. Results are represented as means \pm SD of triplicate experiments. 
FIG 5

A
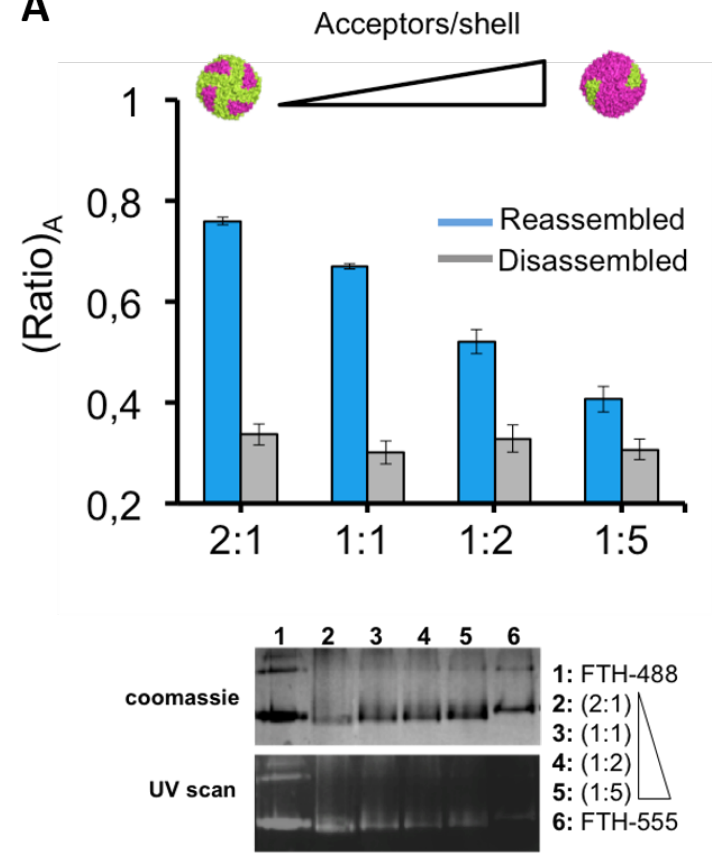

B
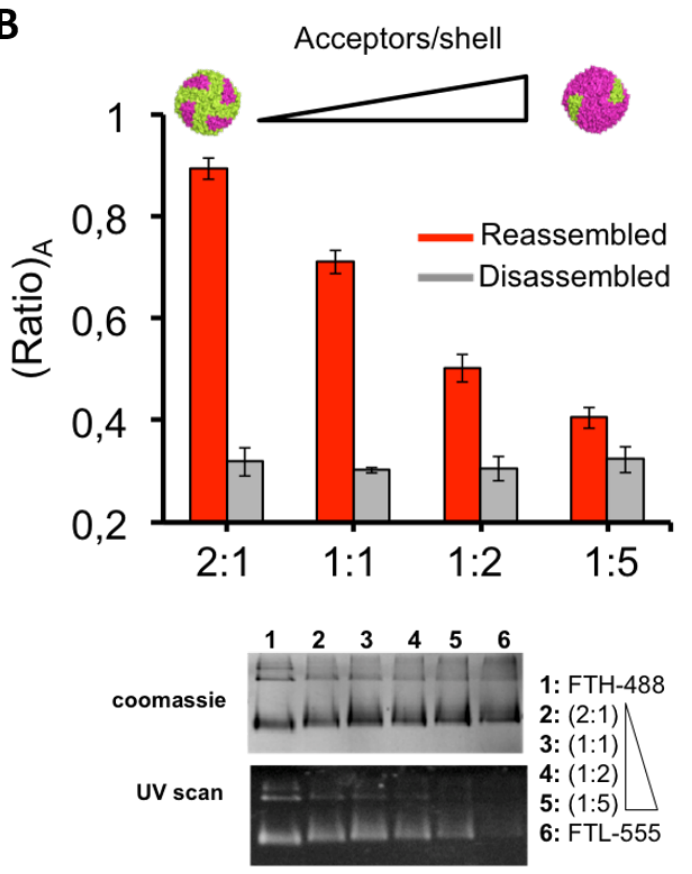

FIGURE 5. FRET properties of isoferritins containing different donor:acceptor ratios. $A$ and $B$, $(\text { ratio })_{\mathrm{A}}$ values of reassembled and disassembled structures of isoferritins containing increasing acceptor ratios. Homopolymeric (blue) and heteropolymeric (red) structures seem to co-assemble with similar efficiency and show a (ratio) A pattern that is a fingerprint of the content of acceptor subunits present on the shell and that is absent on the homolog disassembled mixtures. Lower Part, nondenaturing PAGE of the reassembled full-labeled ferritins containing different donor:acceptor ratios, scanned under UV light and Coomassie stained, which shows a protein ladder in agreement with their subunit composition. Results are represented as means \pm SD of triplicate experiments. 


\section{FIG 6}

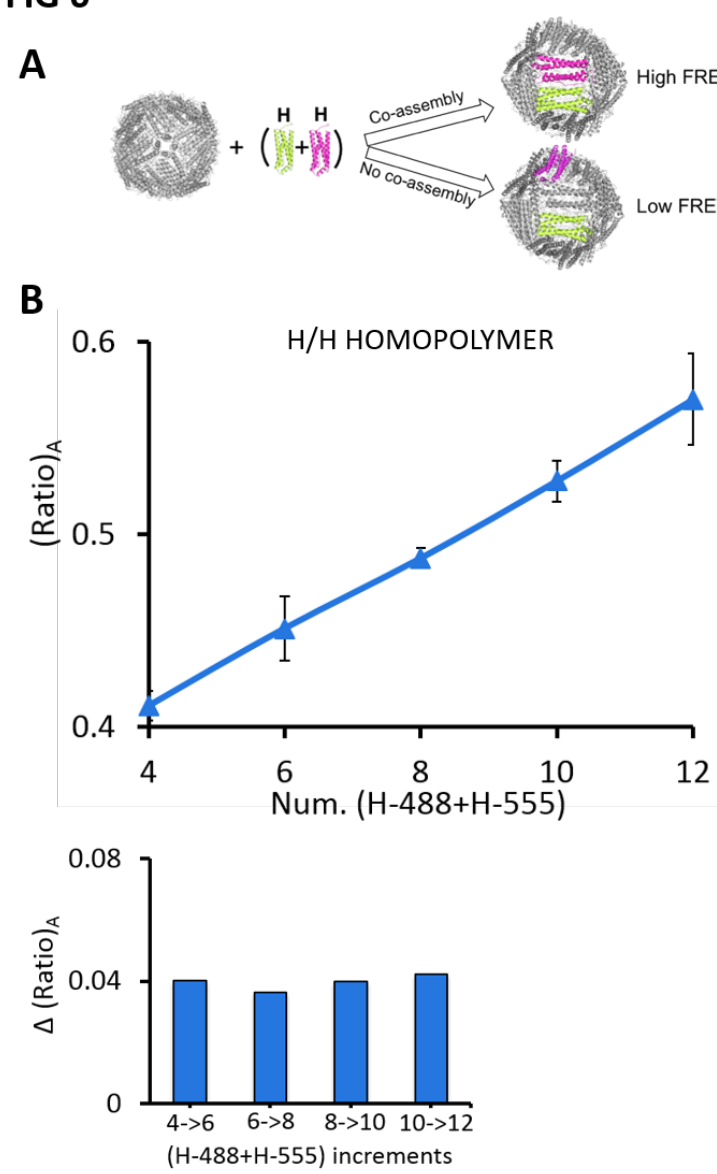

\section{: Unlabelled H or L chain
: Donor H-488
: Acceptor H-555}

C
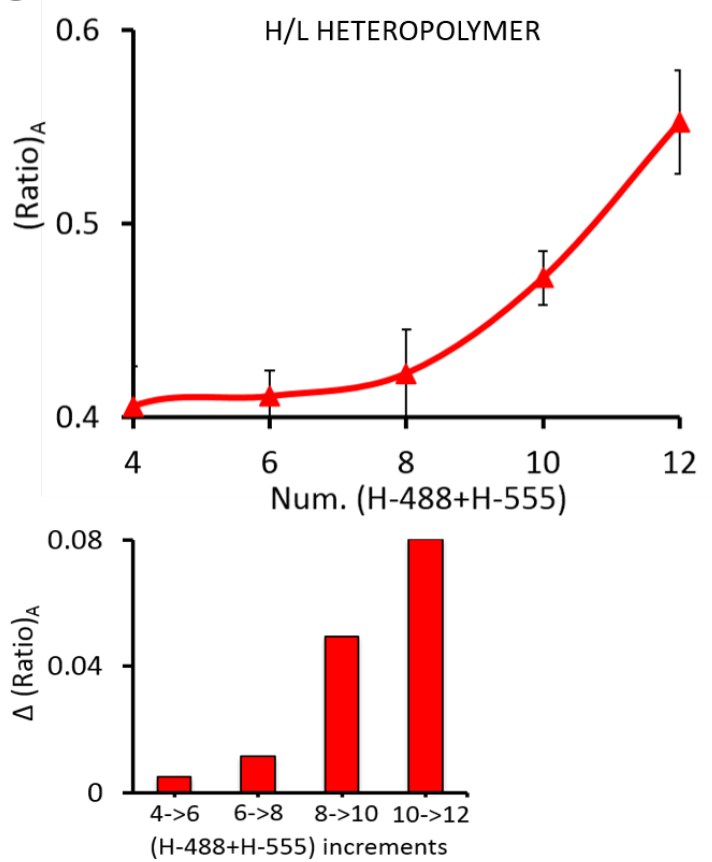

FIGURE 6. Design of a system capable to compare the effect of H- and L-chains in the assembly of the $\mathrm{H}$-chain. $A$, scheme of reaction. A system capable to compare the effect of $\mathrm{H}$ - and $\mathrm{L}$-chains in the assembly of the H-chains was designed. More specifically, we used donor-acceptor H-chain couples (H-488 and H-555, green and magenta structures) as sensors of H-subunit proximity and analyzed how various proportions of unlabeled H- and L-chains (grey structures) interfered with this proximity. An association of the FRET $H$ subunits in the final shell would yield high FRET-efficiencies while

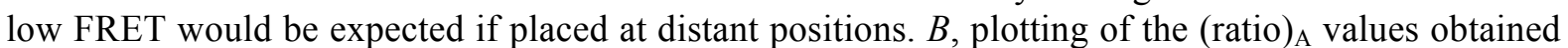
for the assembly of different amounts of FRET couples $(\mathrm{H}-488+\mathrm{H}-555)$ together with unlabeled $\mathrm{H}$ chains. The FRET-efficiency increases linearly with the number of fluorescent $\mathrm{H}$-subunits indicating a random distribution of the fluorescent $\mathrm{H}$-chains not affected by the intervening unlabeled H-chains. Bottom, plotting of the increments in (ratio) ${ }_{\mathrm{A}}$ with respect to the number of fluorescent subunits per shell. $C$, plotting of the (ratio) $)_{\mathrm{A}}$ values obtained for the assembly of different amounts of FRET couples $(\mathrm{H}-488+\mathrm{H}-555)$ together with unlabeled L-chains. Negligible changes in FRET after the addition of 20, 18 and $16 \mathrm{~L}$-chains, and a large increase after $12 \mathrm{~L}$-chains, indicating that the H-chain distribution in the presence of L-chains is not random, but it occupies preferential sites in the 24-mer heteropolymeric shell which tend to separate the FRET couple. Bottom, plotting of the increments in $(\text { ratio })_{A}$ with respect to the number of fluorescent subunits per shell highlighting the strong jump on $(\text { ratio })_{\mathrm{A}}$ when more than $8 \mathrm{H}$-chains/molecule. Results are represented as means $\pm \mathrm{SD}$ of triplicate experiments. 
FIG 7

A

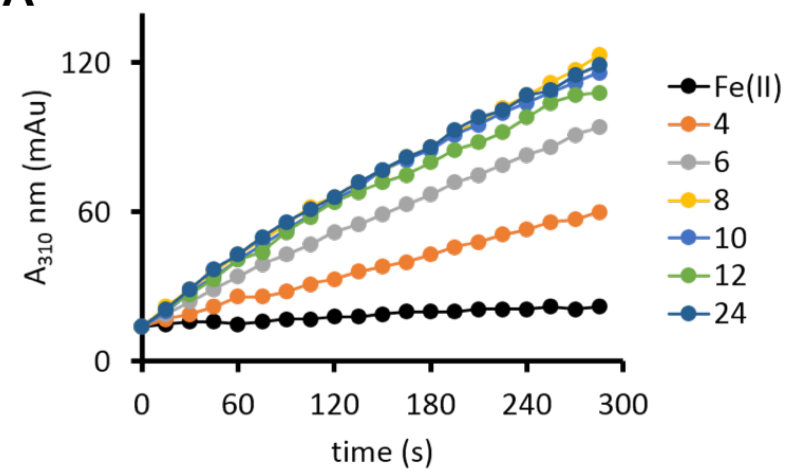

B

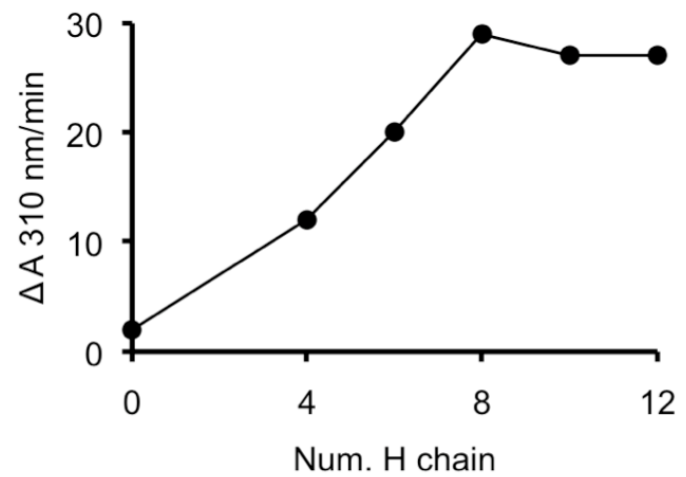

FIGURE 7. $A$. Progression plots of iron uptake monitored by absorbance at $310 \mathrm{~nm}$ of H/L heteropolymers containing a total of 4, 6, 8, 10 and 12 H-chains. Homopolymeric FTH (24 H-chains) and control containing $\mathrm{Fe}(\mathrm{II})$ in the absence of ferritins are also showed. $B$. Initial rates of iron uptake of the ferritins showed in $A$. Conditions: $0.1 \mu \mathrm{M}$ apoferritins, $0.1 \mathrm{M}$ Tris-HCL pH 6.5, $0.1 \mathrm{mM}$ ferrous ammonium sulfate, $25^{\circ} \mathrm{C}$. Results of triplicate experiments are represented as means $\pm \mathrm{SD}$. 